\title{
Rescattering corrections and self-consistent metric in planckian scattering
}

\author{
M. Ciafaloni ${ }^{a}$ and D. Colferai ${ }^{b}$ \\ ${ }^{a}$ c/o Dipartimento di Fisica, Università di Firenze, \\ Via Sansone 1, 50019 Sesto Fiorentino, Italy \\ ${ }^{b}$ Dipartimento di Fisica, Università di Firenze and INFN, Sezione di Firenze, \\ Via Sansone 1, 50019 Sesto Fiorentino, Italy \\ E-mail: ciafaloni@fi.infn.it, colferai@fi.infn.it
}

ABSTRACT: Starting from the ACV approach to transplanckian scattering, we present a development of the reduced-action model in which the (improved) eikonal representation is able to describe particles' motion at large scattering angle and, furthermore, UV-safe (regular) rescattering solutions are found and incorporated in the metric. The resulting particles' shock-waves undergo calculable trajectory shifts and time delays during the scattering process - which turns out to be consistently described by both action and metric, up to relative order $R^{2} / b^{2}$ in the gravitational radius over impact parameter expansion. Some suggestions about the role and the (re)scattering properties of irregular solutions not fully investigated here - are also presented.

KeYwords: Models of Quantum Gravity, Black Holes in String Theory

ARXIV EPRINT: 1406.6540 


\section{Contents}

1 Introduction 1

2 Improved leading eikonal description and particle motion 3

2.1 Eikonal representation of the scattering amplitude 3

$\begin{array}{ll}2.2 & \text { Wave packet motion induced by the } S \text {-matrix }\end{array}$

2.3 Scattering description by trajectory shifts 9

3 Shifted shock-wave fields from the improved eikonal model 13

4 Rescattering corrections to the reduced-action model $\quad 18$

$\begin{array}{lll}4.1 & \text { Semiclassical field equations } & 18\end{array}$

4.2 The reduced-action without rescattering 20

4.3 The H-diagram: scattering angle and shifts 22

$\begin{array}{lll}4.4 & \text { Rescattering solutions with shifted fields } & 25\end{array}$

4.5 Shift modification for the transverse field 28

$\begin{array}{lll}5 & \text { Irregular solutions and their (re)scattering properties } & 29\end{array}$

6 Conclusions $\quad 35$

$\begin{array}{ll}\text { A H-diagram insertions } & 37\end{array}$

\section{Introduction}

Interest in the gravitational $S$-matrix at transplanckian energies [1-7] has revived in the past few-years [8-10], when explicit solutions of the so-called reduced-action model [7] have been found [8]. The model is a much simplified version of the ACV eikonal approach [1$3,6]$ to transplanckian scattering in string-gravity, and is valid in the regime in which the gravitational radius $R \equiv 2 G \sqrt{s}$ is much larger than the string length $\lambda_{s} \equiv \sqrt{\alpha^{\prime} \hbar}$, so that string-effects are supposed to be small.

The reduced-action model (section 4) was derived by justifying the eikonal form of the $S$-matrix at impact parameter $b$ on the basis of string dynamics and by then calculating the eikonal itself (of order $\sim \frac{G s}{\hbar} \gg 1$ ) in the form of a 2-dimensional action, whose power series in $R^{2} / b^{2}$ corresponds to an infinite sum of proper irreducible diagrams (the "multiH" diagrams $[6,7]$ ), evaluated in the high-energy limit. The model admits a quantum generalization [9] of the $S$-matrix in the form of a path-integral — with definite boundary conditions - of the reduced-action exponential itself.

The main feature of the model and of its boundary conditions is the existence of a critical impact parameter $b_{c} \sim R$ such that, for $b>b_{c}$ the $S$-matrix matches the perturbative 
series and is unitary, while for $b<b_{c}$ the field solutions are complex-valued and the elastic $S$-matrix is suppressed exponentially. The suppression exponent is of order $\frac{G s}{\hbar} \sim \frac{R^{2}}{\lambda_{P}^{2}}\left(\lambda_{P}\right.$ being the Planck length) or, if we wish, of the same order as the entropy of a black-hole of radius $R$. From various arguments we believe that in the region in which $b<b_{c}$ (that is, $b$ is smaller than the gravitational radius), a classical gravitational collapse is taking place.

A key issue related to the collapse region is the possible existence of information loss at quantum level, which in the ACV model shows up as lack of $S$-matrix unitarity. According to [9] such unitarity loss is mostly related to a restrictive boundary condition, which is required for the solutions of the model be UV-safe. If such condition is relaxed, further solutions show up, which could contribute to unitarity, but are irregular - i.e., dominated by planckian distances, region in which the model itself is inadequate.

The main motivation of the present paper is to improve and complete some unsatisfactory aspects of the reduced-action model which might be crucial at planckian distances, but show up already at distances of order $R \equiv 4 G E$, the gravitational radius. The new model that we shall present here features two improvements. Firstly, the eikonal representation itself is embedded in three dimensions, so as to be able to describe the motion of the Breitframe during the scattering process. By comparison, the usual two-dimensional representation is inadequate outside the regime of very small angle scattering, and in particular in the collapse region. In fact, the energy-momentum of the impinging particles is taken to be an external light-like source - without any deflection — which generates gravitational fields characterized by Aichelburg-Sexl-like shock waves [11]. However, the resulting action predicts the Einstein deflection angle [1-3] and corrections to it [6], at variance with the original assumption of frozen sources. Instead, we know from the beginning $[12,13]$ that in the classical limit the gravitational equations should predict both particle motion and fields in a self-consistent way. For that to be possible, the improved eikonal representation is needed.

The additional important improvement of the present model is the treatment of rescattering corrections of the produced gravitons. Such effects were argued in [14] to be of particular interest for the irregular solutions of the model, perhaps explaining their relationship to collapse and their contribution to unitarity. But, independently of such a feature, we shall argue here that even for regular (UV-safe) solutions, rescattering corrections are needed in order to achieve self-consistency of motion and metric at higher orders in the eikonal expansion.

To start with, we deal with the self-consistency problem at leading level, in which the eikonal refers to frozen undeflected sources, the metric contains two Aichelburg-Sexl (AS) shock waves, while the action predicts the Einstein deflection angle $\theta_{E}=2 R / b$, as function of the impact parameter $b$ and of the gravitational radius $R(E)=4 G E(2 E=\sqrt{s}$ being the invariant mass of the system). The problem in introducing the particle motion is that the kinematical corrections implementing it are of the same relative order $\left(\theta_{E}^{2}\right.$ or higher) as the irreducible dynamical corrections to the Einstein deflection. How to disentangle the former terms from the latter ones?

A hint about solving the question above was provided in ref. [6], where it was shown that - given the particular Coulombic form of the leading eikonal — it is possible to 
take into account the motion of the Breit-frame of the particles without affecting the $S$ matrix eigenvalue which is still provided by the naive two-dimensional Fourier transform. Such observation is the basis for our treatment of motion in section 2, which suggests writing a modified metric, which is self-consistent at leading level. The main difference with the previous one [8] lies in the introduction of two-body "shifts" of 't Hooft type [15], summarizing the action of the leading $S$-matrix at two-body level.

That is not enough however. The reduced-action model contains the longitudinal fields $h^{++}\left(h^{--}\right)$characterized by AS shock waves centered on the particles with profile functions $2 \pi R a(\boldsymbol{x})(2 \pi R \bar{a}(\boldsymbol{x}))$; furthermore, it generates a gravitational wave also with a field $h=\nabla^{2} \phi$ defined by the H-diagram. The longitudinal field is here calculated in the improved eikonal representation in section 3 and found to be consistent with AS waves [11] which are delayed in time and rotated in space, as suggested in section. 2. The transverse field, on the other hand, provides corrections to the leading eikonal profiles in $a$ and $\bar{a}$, and causes modifications of the metric inside the light-cone by providing important rescattering corrections.

We then set up a perturbative procedure, in order to deal with the self-consistency problem at higher orders in the eikonal expansion. At next order we find the H-diagram deflection [6] which again appears as a feature of the action, but is not incorporated in the metric, which would require further modifications of the energy-momentum tensor. Surprisingly, we find that the class of multi-H diagrams considered in [1-3] is not sufficient in order to provide a satisfactory metric at this level but we need to go one further step, and calculate rescattering corrections, which carry the information due to two-body shifts for the produced gravitons. Such shifts occur in an approximate solution to the rescattering equations under consideration since a long time [16], which is here worked out to completion in section 4 . The results so obtained complete the picture of the improved eikonal representation in section 3 for corrections of relative order $R^{2} / b^{2}$.

Finally, in section 5 we somewhat change subject, and we address the gross features of the ultraviolet-sensitive solutions of the model, whose importance lies in the fact that they could play a role for the recovery of unitarity and/or the related information loss. We then summarize the essential results of the paper and their consequences for action and metric in section 6 , by discussing also some suggestions which arise from our preliminary analysis of singular solutions.

\section{Improved leading eikonal description and particle motion}

\subsection{Eikonal representation of the scattering amplitude}

ACV [6] have shown that the leading contributions to the high-energy elastic scattering amplitude come from the $s$-channel iteration of soft-graviton exchanges, which can be represented by effective ladder diagrams as in figure 1. The purpose of the present section is to recall the method of resumming the effective ladder contributions to all orders so as to provide the so-called eikonal representation for the elastic $S$ matrix. This representation is here "improved" in the sense that we do not make a separation of longitudinal and transverse variables by neglecting the leading scattering angle. Instead, by following [6], we use the exact 3-dimensional phase-space of the on-shell particles at each gravitational 


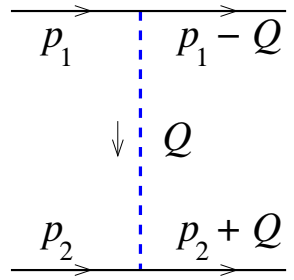

(a)

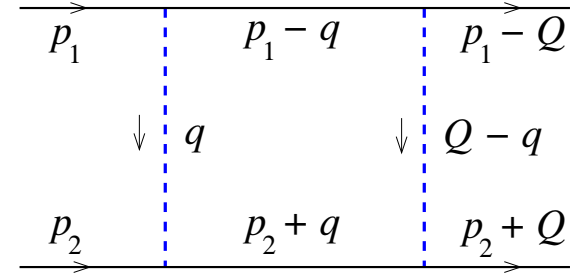

(b)

Figure 1. One- and two-rung effective ladder diagrams determining the elastic $S$-matrix in the eikonal approximation. Solid lines: on-shell external particles; dashed lines: eikonal gravitational exchanges.

eikonal exchange and we prove that — due to the Coulombic form of the exchange - the $S$-matrix is nevertheless provided by a 2 -dimensional transform.

It is important to note that the exchanged gravitons (dashed lines) are associated to a propagator $-\mathrm{i} / Q^{2}$ and are coupled to the colliding particles/strings (solid lines) with an interaction strength equal to $\alpha \equiv G s / \hbar$ for each pair of vertices. Furthermore, the particles' lines are on-shell along the effective ladder, as proved in ref [1-3] by the sum over inelastic excitations also. The generic ladder is thus built by iteration of the basic rung

$$
\mathcal{R}_{1}\left(p_{1}, p_{2}, Q\right)=\mathrm{i} \mathcal{M}_{1}\left(Q^{2}, s\right) 2 \pi \delta_{+}\left(\left(p_{1}-Q\right)^{2}\right) 2 \pi \delta_{+}\left(\left(p_{2}+Q\right)^{2}\right), \quad \mathcal{M}_{1}\left(Q^{2}, s\right) \equiv-\frac{8 \pi G s^{2}}{Q^{2}}
$$

We start computing the 2-rung ladder (figure 1.b)

$$
\begin{aligned}
\mathcal{R}_{2}\left(p_{1}, p_{2}, Q\right) & =\int \frac{\mathrm{d}^{4} q}{(2 \pi)^{4}} \mathcal{R}_{1}\left(p_{1}, p_{2}, q\right) \mathcal{R}_{1}\left(p_{1}-q, p_{2}+q, Q-q\right) \\
& =\mathrm{i}^{2} \mathcal{M}_{2}\left(Q^{2}, s\right) 2 \pi \delta_{+}\left(\left(p_{1}-Q\right)^{2}\right) 2 \pi \delta_{+}\left(\left(p_{2}+Q\right)^{2}\right) \\
\mathcal{M}_{2}\left(Q^{2}, s\right) & \equiv \int \frac{\mathrm{d}^{4} q}{(2 \pi)^{2}} \mathcal{M}_{1}\left(q^{2}, s\right) \mathcal{M}_{1}\left((Q-q)^{2}, s\right) \delta_{+}\left(\left(p_{1}-q\right)^{2}\right) \delta_{+}\left(\left(p_{2}+q\right)^{2}\right) .
\end{aligned}
$$

In the center-of-mass $(\mathrm{CM})$ frame where $p_{1}=(E, 0,0, E), p_{2}=(E, 0,0,-E), Q=\left(Q^{0}, \vec{Q}\right)$, $s=4 E^{2}$, the two mass-shell delta functions in eq. (2.3) become

$$
\delta_{+}\left(\left(p_{1}-q\right)^{2}\right) \delta_{+}\left(\left(p_{2}+q\right)^{2}\right)=\frac{1}{2 s|\vec{q}|} \delta\left(q^{0}\right) \delta\left(\cos \theta-\frac{|\vec{q}|}{2 E}\right), \quad\left(\cos \theta=\hat{p}_{1} \cdot \hat{q}\right)
$$

and, for the same reason, the two external mass-shell deltas in eq. (2.2) constrain $Q^{0}=$ $0, \cos \theta_{Q} \equiv \hat{p}_{1} \cdot \hat{Q}=|\vec{Q}| / 2 E, Q^{2}=-\vec{Q}^{2}$. In this way, the $4 \mathrm{D}$ integration $\mathrm{d}^{4} q=$ $\mathrm{d} q^{0}|\vec{q}|^{2} \mathrm{~d}|\vec{q}| \mathrm{d} \cos \theta \mathrm{d} \phi$ reduces to a $2 \mathrm{D}$ integral $^{1}$

$$
\mathcal{M}_{2}=\int \frac{|\vec{q}| \mathrm{d}|\vec{q}| \mathrm{d} \phi}{(2 \pi)^{2}} \mathcal{M}_{1}\left(-\vec{q}^{2}, s\right) \frac{4 \pi G s}{(\vec{Q}-\vec{q})^{2}}
$$

\footnotetext{
${ }^{1}$ This shows that $\mathcal{M}_{2}$ is a function of $s$ and $Q^{2}$.
} 
By taking into account the expression of $\cos \theta$ and $\cos \theta_{Q}$ in terms of $|\vec{q}|,|\vec{Q}|$ and $E$ and using the identity

$$
\int_{0}^{2 \pi} \frac{\mathrm{d} \phi}{a+b \cos \phi}=\frac{2 \pi}{\sqrt{a^{2}-b^{2}}}
$$

it is easy to evaluate the azimuthal integral in eq. (2.5) and, more interestingly, to show that it can be rewritten as the azimuthal integral of a pure $2 \mathrm{D}$ propagator:

$$
\begin{aligned}
& \int_{0}^{2 \pi} \frac{\mathrm{d} \phi}{(\vec{Q}-\vec{q})^{2}}=\int_{0}^{2 \pi} \frac{\mathrm{d} \phi}{\vec{Q}^{2}+\vec{q}^{2}-2|\vec{Q} \| \vec{q}|\left(\cos \theta \cos \theta_{Q}-\sin \theta \sin \theta_{Q} \cos \left(\phi-\phi_{Q}\right)\right)} \\
& =\frac{2 \pi}{\sqrt{\left(\vec{Q}^{2}+\vec{q}^{2}\right)^{2}-4 \vec{Q}^{2} \vec{q}^{2}}}=\int_{0}^{2 \pi} \frac{\mathrm{d} \phi^{\prime}}{\vec{Q}^{2}+\vec{q}^{2}-2|\vec{Q} \| \vec{q}| \cos \phi^{\prime}}=\int_{0}^{2 \pi} \frac{\mathrm{d} \phi^{\prime}}{(\boldsymbol{Q}-\boldsymbol{q})^{2}}
\end{aligned}
$$

where $\boldsymbol{Q}$ and $\boldsymbol{q}$ are fictitious 2D vectors with the same modulus as $\vec{Q}$ and $\vec{q}$ respectively, and $\phi^{\prime}$ is the angle between them. With this trick, $\mathcal{M}_{2}$ can be rewritten as a $2 \mathrm{D}$ convolution:

$$
\mathcal{M}_{2}\left(Q^{2}, s\right)=\int \frac{\mathrm{d}^{2} \boldsymbol{q}}{(2 \pi)^{2}} \mathcal{M}_{1}\left(-\boldsymbol{q}^{2}, s\right) \frac{4 \pi G s}{(\boldsymbol{Q}-\boldsymbol{q})^{2}}=\frac{1}{2 s}\left[\mathcal{M}_{1} \otimes \mathcal{M}_{1}\right](\boldsymbol{Q}), \quad\left(\boldsymbol{Q}^{2}=-Q^{2}\right) .
$$

It is now straightforward to generalize that procedure to the $n$-rung ladder, by deriving the recursion formula

$$
\begin{aligned}
\mathcal{R}_{n}\left(p_{1}, p_{2}, Q\right) & =\int \frac{\mathrm{d}^{4} q}{(2 \pi)^{4}} \mathcal{R}_{n-1}\left(p_{1}, p_{2}, q\right) \mathcal{R}_{1}\left(p_{1}-q, p_{2}+q, Q-q\right) \\
& =\mathrm{i}^{n} \mathcal{M}_{n}\left(Q^{2}, s\right) 2 \pi \delta_{+}\left(\left(p_{1}-Q\right)^{2}\right) 2 \pi \delta_{+}\left(\left(p_{2}+Q\right)^{2}\right) \\
\mathcal{M}_{n}\left(Q^{2}, s\right) & =\int \frac{\mathrm{d}^{2} \boldsymbol{q}}{(2 \pi)^{2}} \mathcal{M}_{n-1}\left(-\boldsymbol{q}^{2}, s\right) \frac{4 \pi G s}{(\boldsymbol{Q}-\boldsymbol{q})^{2}}=2 s\left[\stackrel{n}{\otimes} \frac{4 \pi G s}{\boldsymbol{Q}^{2}}\right] .
\end{aligned}
$$

It should be noted that such simplification is due to the peculiar form of the graviton interaction from which eq. (2.7) follows.

Finally, the eikonal $S$ matrix (with overall momentum conservation factored out) is given by the sum of the ladder diagrams with the usual combinatorial factor $1 / n$ ! and with the final-state mass-shell deltas removed:

$$
S_{\text {eik }}\left(Q^{2}, s\right)=\frac{1}{2 s} \sum_{n=0}^{\infty} \frac{\mathrm{i}^{n}}{n !} \mathcal{M}_{n}\left(Q^{2}, s\right)=\sum_{n=0}^{\infty} \frac{(\mathrm{i} 4 \pi G s)^{n}}{n !}\left[\stackrel{n}{\otimes} \frac{1}{Q^{2}}\right] .
$$

By applying a 2D Fourier transform to eq. (2.11a), convolutions become standard products and the r.h.s. of eq. (2.11a) reduces to an exponential series:

$$
\tilde{S}_{\text {eik }}(\boldsymbol{b}, s) \equiv \int \frac{\mathrm{d}^{2} \boldsymbol{Q}}{(2 \pi)^{2}} \mathrm{e}^{-\mathrm{i} \boldsymbol{Q} \cdot \boldsymbol{b}} S_{\text {eik }}\left(-\boldsymbol{Q}^{2}, s\right)=\exp \left\{\mathrm{i} A_{\text {eik }}(\boldsymbol{b}, s)\right\}, \quad A_{\text {eik }} \equiv \tilde{\mathcal{M}}_{1}
$$

where we interpret the $2 \mathrm{D}$ Fourier transform of the one-graviton amplitude $\tilde{\mathcal{M}}_{1}$ as eikonal action $A_{\text {eik }}$. Actually, the definition of $A_{\text {eik }}$ (and of all convolutions involved in this calculation) requires the introduction of an infrared cutoff $Q_{0} \sim 1 / L$ in order to regularize the "Coulomb" divergence typical of long-range interactions $\sim 1 / Q^{2}$ :

$$
A_{\text {eik }}(\boldsymbol{b}, s) \equiv 2 \delta_{0}(b, s)=\int \frac{\mathrm{d}^{2} \boldsymbol{Q}}{(2 \pi)^{2}} \mathrm{e}^{-\mathrm{i} \boldsymbol{Q} \cdot \boldsymbol{b}} \frac{4 \pi G s}{\boldsymbol{Q}^{2}} \Theta\left(\boldsymbol{Q}^{2}-Q_{0}^{2}\right)=2 G s \ln \frac{L}{b}+\mathcal{O}\left(\frac{b}{L}\right)^{2}
$$


where $b \equiv|\boldsymbol{b}|$ and $L \equiv 2 \mathrm{e}^{-\gamma_{E}} / Q_{0}$.

A couple of remarks are in order:

- The "position" variable $\boldsymbol{b}$, being conjugate to $\boldsymbol{Q}$, is usually interpreted as impact parameter of the collision, and $\delta_{0}(b, s)$ as the associated phase shift. However, $\boldsymbol{Q}$ is a fictitious $2 \mathrm{D}$ vector whose polar angle is actually undefined, therefore $\boldsymbol{b}$ cannot be thought of as a purely transverse (to $\vec{p}_{1}$ ) vector.

- The dependence of $\tilde{S}$ on $L$ amounts to a $b$-independent phase, and therefore it is unimportant in the determination of the scattering angle. However such a phase depends on the total energy $\sqrt{s}$. This may cause a cutoff-dependent time evolution of the scattered particles, hopefully without physical consequences.

Both issues will be discussed in detail in the next section.

\subsection{Wave packet motion induced by the $S$-matrix}

The purpose of this section is to derive the motion of two quantum particles subject to a scattering amplitude given by the leading eikonal result of [6] and recalled in section 2.1. Suppose we prepare a state of two well separated light-like free particles in the past. In a first-quantization description ${ }^{2}$ the wave function is given by

$$
\psi_{\text {in }}\left(t, \vec{x}_{1}, \vec{x}_{2}\right)=\int \mathrm{d}^{3} p_{1} \mathrm{~d}^{3} p_{2} \tilde{\psi}\left(\vec{p}_{1}, \vec{p}_{2}\right) \mathrm{e}^{-\mathrm{i} E_{1} t+\mathrm{i} \vec{p}_{1} \cdot \vec{x}_{1}} \mathrm{e}^{-\mathrm{i} E_{2} t+\mathrm{i} \vec{p}_{2} \cdot \vec{x}_{2}}, \quad\left(E_{i}=\left|\vec{p}_{i}\right|\right) .
$$

In the far future the wave function is expressed in terms of the $S$-matrix as follows:

$$
\begin{aligned}
\psi_{\text {out }}\left(t, \vec{x}_{1}, \vec{x}_{2}\right) & =\int \mathrm{d}^{3} p_{1} \mathrm{~d}^{3} p_{2} \mathrm{~d}^{3} k_{1} \mathrm{~d}^{3} k_{2} \tilde{\psi}\left(\vec{p}_{1}, \vec{p}_{2}\right)\left\langle\vec{k}_{1}, \vec{k}_{2}|S| \vec{p}_{1}, \vec{p}_{2}\right\rangle \mathrm{e}^{-\mathrm{i} k_{1} x_{1}} \mathrm{e}^{-\mathrm{i} k_{2} x_{2}} \\
k_{j} & =\left(E_{j}^{\prime}, \vec{k}_{j}\right), \quad E_{j}^{\prime}=\left|\vec{k}_{j}\right| .
\end{aligned}
$$

It is convenient to perform the calculation in the CM frame. As usual, we define the CM coordinate $\vec{X}$ and relative coordinate $\vec{x}$

$$
\begin{aligned}
& \vec{X} \equiv \frac{\vec{x}_{1}+\vec{x}_{2}}{2} \quad \text { conjugated to total momentum } \quad \vec{P} \equiv \vec{p}_{1}+\vec{p}_{2} \\
& \vec{x} \equiv \vec{x}_{1}-\vec{x}_{2} \quad \text { conjugated to relative momentum } \quad \vec{p} \equiv \frac{\vec{p}_{1}-\vec{p}_{2}}{2}
\end{aligned}
$$

For simplicity, we limit ourselves to states which have zero total momentum, by setting

$$
\tilde{\psi}\left(\vec{p}_{1}, \vec{p}_{2}\right)=\delta^{3}\left(\vec{p}_{1}+\vec{p}_{2}\right) \tilde{\psi}(\vec{p})
$$

With this restriction the CM coordinate is completely delocalized, but we can build a state representing a localized wave packet in the relative coordinate:

$$
\tilde{\psi}(\vec{p})=\tilde{N} \exp \left\{-\frac{1}{2}\left(\frac{\vec{p}-\vec{p}_{0}}{\sigma}\right)^{2}-\mathrm{i} \vec{p} \cdot \vec{x}_{0}+\mathrm{i} 2 E_{p} t_{0}\right\},
$$

\footnotetext{
${ }^{2}$ Since we are neglecting particle production, the first-quantization framework is suited for our purposes.
} 
where $\vec{p}=\vec{p}_{1}=-\vec{p}_{2}$ is the relative momentum, $\vec{p}_{0}=(0,0, E)$ is its mean value and $\sigma$ a measure of its broadening, $\tilde{N}$ is a normalization factor, $2 E_{p}=2|\vec{p}|$ is the total CM energy, while $\vec{x}_{0}$ is the relative position at time $t_{0}$. In fact, by inserting eqs. (2.16) into eq. (2.13) we are left with an integral in $\vec{p}$ whose phase $\Phi_{\text {in }}=-2 E_{p}\left(t-t_{0}\right)+\vec{p} \cdot\left(\vec{x}-\vec{x}_{0}\right)$ is stationary for

$$
\vec{x}_{\mathrm{in}}-\vec{x}_{0}=2 \nabla_{\vec{p}} E_{p}\left(t-t_{0}\right) \simeq 2 \frac{\vec{p}_{0}}{E}\left(t-t_{0}\right)
$$

since the shape of $\tilde{\psi}$ forces $\vec{p} \simeq \vec{p}_{0}$. Going back to particles' coordinates, we confirm that in the past each particle moved at the speed of light in the direction of $\vec{p}_{0}$ :

$$
\vec{x}_{1}(t)=-\vec{x}_{2}(t)=\frac{\vec{x}}{2}=\frac{1}{2} \vec{x}_{0}+\hat{p}_{0}\left(t-t_{0}\right), \quad \hat{p}_{0} \equiv \frac{\vec{p}_{0}}{E}=(0,0,1) .
$$

On the other hand, by substituting eqs. (2.16) in the outgoing wave (2.14) together with the energy-momentum conserving eikonal $S$-matrix (2.11)

$$
\begin{aligned}
\left\langle\vec{k}_{1}, \vec{k}_{2}|S| \vec{p}_{1}, \vec{p}_{2}\right\rangle & =\delta^{4}\left(k_{1}+k_{2}-p_{1}-p_{2}\right) 2 s \int \mathrm{d}^{2} \boldsymbol{b} \mathrm{e}^{i \boldsymbol{Q} \cdot \boldsymbol{b}+i A_{\mathrm{eik}}(\boldsymbol{b}, s)} \\
Q & \equiv p_{1}-k_{1}=p-k, \quad \boldsymbol{Q}^{2} \equiv-Q^{2}, \quad s=\left(2 E_{p}\right)^{2}
\end{aligned}
$$

we find

$$
\begin{aligned}
\psi_{\text {out }} & =\frac{1}{2} \tilde{N} \int \mathrm{d}^{3} p \mathrm{~d}^{3} k \mathrm{~d}^{2} \boldsymbol{b} \delta\left(E_{k}-E_{p}\right) \exp \left\{-\frac{1}{2}\left(\frac{\vec{p}-\vec{p}_{0}}{\sigma}\right)^{2}\right\} \exp \left(\mathrm{i} \Phi_{\text {out }}\right) \\
\Phi_{\text {out }} & =-2 E_{p}\left(t-t_{0}\right)+\vec{k} \cdot \vec{x}-\vec{p} \cdot \vec{x}_{0}+\boldsymbol{Q} \cdot \boldsymbol{b}+2 G s \log \frac{L}{b}
\end{aligned}
$$

where $\boldsymbol{Q}$ and $\boldsymbol{b}$ are auxiliary 2D vectors, the former being determined by the condition $Q^{2}=-Q^{2}$. In this case, the stationarity of $\Phi_{\text {out }}$ with respect to independent variations of $\vec{p}, \vec{k}$ and $\boldsymbol{b}$ yields the asymptotic motion in the future, as follows. In the plane of scattering $\langle\vec{p}, \vec{k}\rangle$ we introduce the polar angles $\theta_{i}$ of momenta and coordinates, and write

$$
\vec{k} \cdot \vec{x}=E_{p} r \cos \theta_{k x}, \quad \vec{p} \cdot \vec{x}_{0}=E_{p} r_{0} \cos \theta_{p x_{0}}, \quad \boldsymbol{Q} \cdot \boldsymbol{b}=|\vec{Q}| b \cos \phi^{\prime}=2 E_{p}\left|\sin \frac{\theta_{k p}}{2}\right| b \cos \phi^{\prime}
$$

where we used the notation $\theta_{i j} \equiv \theta_{i}-\theta_{j}, b \equiv|\boldsymbol{b}|, r \equiv|\vec{x}|$ and the relations $E_{k}=E_{p}$, $|\vec{Q}|=2 E_{p}\left|\sin \left(\theta_{k p} / 2\right)\right|$.

We can now determine the five unknowns $r, \theta_{k}, \theta_{p}$ and $\boldsymbol{b}$ by finding the stationarity point of the phase with respect to variations of the five integration variables $E_{p}, \theta_{k}, \theta_{p}$ and $\boldsymbol{b}$, while $t_{0}$ and $\vec{x}_{0}$ are known parameters. We have

$$
\begin{aligned}
& 0=\frac{\partial \Phi_{\text {out }}}{\partial \boldsymbol{b}}=\boldsymbol{Q}+\frac{\partial A_{\mathrm{eik}}}{\partial \boldsymbol{b}}=\boldsymbol{Q}-\hat{b} \frac{2 G s}{b} \\
& 0=\frac{\partial \Phi_{\mathrm{out}}}{\partial \theta_{p}}=E_{p} r_{0} \sin \theta_{p x_{0}}-\epsilon\left(\theta_{k p}\right) E_{p} b \cos \frac{\theta_{k p}}{2} \cos \phi^{\prime} \\
& 0=\frac{\partial \Phi_{\mathrm{out}}}{\partial \theta_{k}}=-E_{p} r \sin \theta_{k x}+\epsilon\left(\theta_{k p}\right) E_{p} b \cos \frac{\theta_{k p}}{2} \cos \phi^{\prime} \\
& 0=\frac{\partial \Phi_{\mathrm{out}}}{\partial E_{p}}=-2\left(t-t_{0}\right)+r \cos \theta_{k x}-r_{0} \cos \theta_{p x_{0}}+2\left|\sin \frac{\theta_{k p}}{2}\right| b \cos \phi^{\prime}+8 E_{p} \frac{\partial A_{\mathrm{eik}}}{\partial s}
\end{aligned}
$$




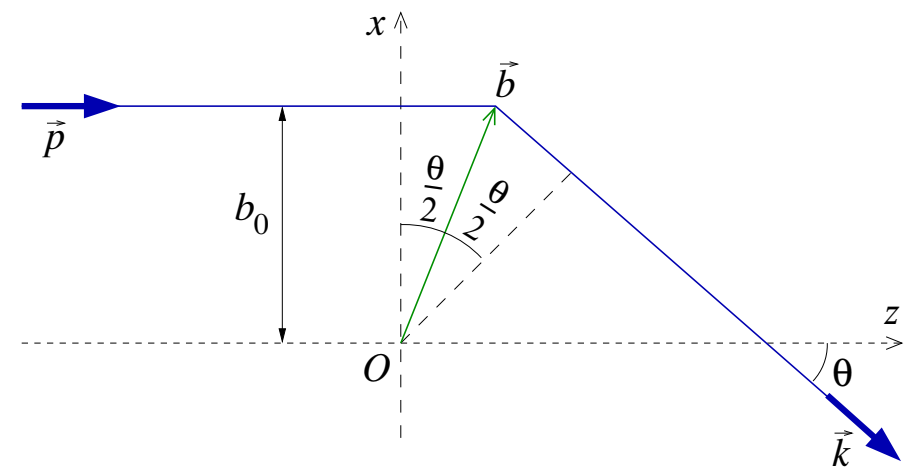

Figure 2. Kinematics of the wave packets' relative coordinates.

where $\epsilon(x)$ denotes the sign of $x$. The first equation causes $\boldsymbol{b}$ to be aligned with $\vec{Q}\left(\phi^{\prime}=0\right)$ and to have modulus

$$
b=\frac{2 G s}{|\vec{Q}|}=\frac{R}{\left|\sin \frac{\theta_{k p}}{2}\right|} .
$$

The second equation yields a further relation involving the physical impact parameter $b_{0}$ :

$$
b_{0} \equiv r_{0} \sin \theta_{x_{0} p}=-\epsilon\left(\theta_{k p}\right) b \cos \frac{\theta_{k p}}{2}=b \cos \frac{\theta}{2}
$$

allowing us to determine the (attractive) scattering angle $\theta \equiv-\theta_{k p}>0$

$$
\tan \frac{\theta}{2}=\frac{R}{b_{0}} .
$$

The third equation identifies the outgoing trajectory to be the straight line

$$
r \sin \theta_{x k}=b \cos \frac{\theta}{2}=b_{0}
$$

with the same impact parameter of the incoming one, thus implying angular momentum conservation. Note that this trajectory intersects the incoming one at the point $\vec{b}=b(\cos \theta / 2,0, \sin \theta / 2))$, as is apparent from figure 2 . The fourth equation provides the motion of the outgoing relative coordinate

$$
\frac{1}{2} r \cos \theta_{x k}=\left(t-t_{0}\right)+\frac{1}{2} r_{0} \cos \theta_{x_{0} p}-b \sin \frac{\theta}{2}-2 R \ln \frac{L}{b}
$$

meaning that each particle keeps on moving at the speed of light.

A very peculiar behaviour of our result is that the motion of the outgoing particles suffers a time delay given by the energy-derivative of the action

$$
t_{\text {delay }}=\frac{1}{2} \frac{\partial A_{\text {eik }}}{\partial E_{p}}=2 R \ln \frac{L}{b}
$$

with respect to a motion with the same deflection but at constant speed. This is easily seen by projecting the incoming trajectory $(2.17)$ on $\vec{p}$

$$
\frac{1}{2} r_{\text {in }} \cos \theta_{x p}=\frac{1}{2} r_{0} \cos \theta_{x_{0} p}+\left(t-t_{0}\right), \quad r_{\text {in }} \sin \theta_{x p}=b_{0}
$$


and by choosing the origin of time $t_{0}=0$ when $\vec{x}_{\text {in }}$ is (or would be) at the intersection point $\vec{b}$ of the trajectories (2.31), (2.34), i.e. $r_{0} \cos \theta_{x_{0} p}=b \sin (\theta / 2)$, so that eqs. (2.34), (2.32) become

$$
\begin{aligned}
\frac{1}{2} r_{\text {in }} \cos \theta_{x p} & =t+\frac{b}{2} \sin \frac{\theta}{2} \\
\frac{1}{2} r_{\text {out }} \cos \theta_{x k} & =t-t_{\text {delay }}-\frac{b}{2} \sin \frac{\theta}{2}
\end{aligned}
$$

showing that $\vec{x}_{\text {out }}=\vec{b}$ at $t=t_{\text {delay. }}$. Let us stress that this time delay is peculiar of gravity, since the energy dependence of the amplitude stems from the gravitational coupling $\alpha=G s$. In other theories, like electrodynamics, the coupling is independent ${ }^{3}$ of the particles' energies, and one obtains analogous equations with $t_{\text {delay }}=0$.

\subsection{Scattering description by trajectory shifts}

We have seen that the leading eikonal $S$-matrix provides a definite scattering angle and wave-packet motion. It also provides a gravitational metric, associated to the gravitaional fields $h_{\mu \nu}=g_{\mu \nu}-\eta_{\mu \nu}$, whose longitudinal terms are expressed in terms of the eikonal amplitude $a(\boldsymbol{x})$ as follows [8]

$$
\begin{array}{ll}
\frac{1}{4} h^{++}=h_{--}=2 \pi R a(\boldsymbol{x}) \delta\left(x^{-}\right), & x^{+} \equiv t+z \\
\frac{1}{4} h^{--}=h_{++}=2 \pi R \bar{a}(\boldsymbol{x}) \delta\left(x^{+}\right), & x^{-} \equiv t-z
\end{array}
$$

where

$$
a(\boldsymbol{x})=\frac{1}{2 \pi} \ln \frac{L^{2}}{\boldsymbol{x}^{2}}, \quad \bar{a}(\boldsymbol{x})=a(\boldsymbol{b}-\boldsymbol{x})
$$

and we actually deal with the situation before collision $(t<0)$.

The collision process, according to the reduced action model to be described in section 4, introduces further (transverse) components of the metric field, and modifies the expressions (2.37) by corrections of relative order $R^{2} / b^{2}$ and higher. Nevertheless, at leading level, starting from the (improved) eikonal representation only, it should be possible to describe the scattering on the basis of an associated metric. Here we suggest a simple way to do it, which is based on 't Hooft's understanding [15] of the $S$-matrix as a coordinate shift.

The starting point is to write the leading order metric before collision, provided by the expressions (2.37) as follows:

$$
\mathrm{d} s^{2}=\mathrm{d} \boldsymbol{x}^{2}-\mathrm{d} x^{-} \mathrm{d} x^{+}+2 \pi R\left[a(\boldsymbol{x}) \delta\left(x^{-}\right)\left(\mathrm{d} x^{-}\right)^{2}+\bar{a}(\boldsymbol{x}) \delta\left(x^{+}\right)\left(\mathrm{d} x^{+}\right)^{2}\right] .
$$

We see that it consists of two Aichelburg-Sexl (AS) shock waves [11] travelling against each other. If only one is present - the one at $x^{+}=0$, say - , a test particle impinging on it will acquire a shift in the $x^{-}$direction:

$$
\Delta x^{-}=2 \pi R \bar{a}(\boldsymbol{x}), \quad \Delta x^{+}=0, \quad \Longrightarrow \quad \Delta t=-\Delta z=\pi R \bar{a}(\boldsymbol{x}) .
$$

\footnotetext{
${ }^{3}$ Or very weakly dependent after renormalization.
} 
As a consequence, after the shift, the test particle will be deflected. In particular, if the particle was moving at fixed $x^{-}$and $\boldsymbol{x}$, the scattering angle $\theta(\boldsymbol{x})$ is given by $\tan (\theta / 2)=$ $\pi R|\nabla \bar{a}(\boldsymbol{x})|$, see figure 2 .

The scattering angle formula is derived by either calculating the action $2 \pi G s \bar{a}(\boldsymbol{x})$ of eq. (2.12) from the shift (2.40) as in ref. [8] and then using eq. (2.30), or by computing the geodesics by standard methods.

The next question is: what happens when both AS waves are present? In order to understand that, consider a test particle at $x^{-}=-\varepsilon<0$, travelling along with particle $1\left(x^{-}=0\right)$ shortly before it. Then, at $x^{+}=0(t=-z=-\varepsilon / 2)$ the test particle will collide with particle 2 and will acquire the shift (2.40), while the location of particle 1 will be forced to do the same with $\Delta x_{1}^{-}=2 \pi R \bar{a}(0)$, evaluated at the "on shell" point $\boldsymbol{x} \equiv \boldsymbol{b}_{0} / 2+\xi=0\left(\xi=-\boldsymbol{b}_{0} / 2\right)$, see figure 3 . Next, the test particle trajectory is deflected on a trajectory $z \simeq t \cos \theta(x), x \simeq-t \sin \theta(x)$, therefore, due to the $\cos \theta$ factor, it will be shortly hit by particle 1 , travelling at $t-z=$ const, and thus will acquire the shift

$$
\Delta x^{+}=2 \pi R a(x), \quad \Delta x^{-}=0 \quad \Longrightarrow \quad \Delta t=\Delta z=\pi R a(x) .
$$

To sum up, the test particle acquires two shifts

$$
\Delta x^{-}=2 \pi R \bar{a}(x) \quad \text { at } \quad x^{+}=0, \quad \Delta x^{+}=2 \pi R a(x) \quad \text { at } \quad x^{-}=2 \pi R \bar{a}(x)
$$

separated by a short time interval $\mathcal{O}(\varepsilon)$, which vanishes in the $\varepsilon \rightarrow 0$ limit. As a consequence, it is natural to assume that particle 1 acquires two shifts also:

$$
\Delta x_{1}^{-}=2 \pi R \bar{a}(0) \quad \text { at } \quad x^{+}=0, \quad \Delta x_{1}^{+}=2 \pi R \tilde{a} \quad \text { at } \quad x^{-}=2 \pi R \bar{a}(0),
$$

where $\tilde{a} \equiv a(0)$ is actually a possibly singular parameter describing the scattering of particle 1 on itself, which is here taken to be a $b_{0}$-independent constant, $\tilde{a}>\bar{a}(0)$.

Taking into account both shifts, particle 1 goes from $z=0^{-}$to $\Delta z=\pi R(\tilde{a}-\bar{a})$ at time $\Delta t=\pi R(\tilde{a}+\bar{a})=2 \pi R \bar{a}+\Delta z \equiv t_{D}+\Delta z$ and is then deflected by an angle $\theta(\boldsymbol{x}=0)$ on the trajectory ${ }^{4}$

$$
\begin{aligned}
& z_{R}(t)=\Delta z \cos \theta-\xi \sin \theta+(t-\Delta t) \\
& x_{R}(t)=\xi \cos \theta+\Delta z \sin \theta=\text { const }
\end{aligned}
$$

where we recall that $x=b_{0} / 2+\xi$. The total collision time - from $z=0^{-}$to $z_{R}\left(t_{C}\right)=0$ - is therefore

$t_{C}=\pi R(\tilde{a}+\bar{a})-\Delta z \cos \theta+\xi \sin \theta=2 \cos ^{2}\left(\frac{\theta}{2}\right) \bar{a}(x)+2 \sin ^{2}\left(\frac{\theta}{2}\right) \tilde{a}+2 \sin \left(\frac{\theta}{2}\right) \cos \left(\frac{\theta}{2}\right) \xi$.

From the stationarity condition of the collision time with respect to $\xi$, we obtain the scattering angle

$$
\tan \frac{\theta(x)}{2}=\pi R\left|\frac{\mathrm{d} \bar{a}(x)}{\mathrm{d} x}\right|_{x=0}=\pi R\left|\frac{\mathrm{d} a\left(b_{0}-x\right)}{\mathrm{d} x}\right|_{x=0}=\frac{R}{b_{0}},
$$

\footnotetext{
${ }^{4}$ The subscript $R$ means "rotated"; see also eqs. (2.55).
} 

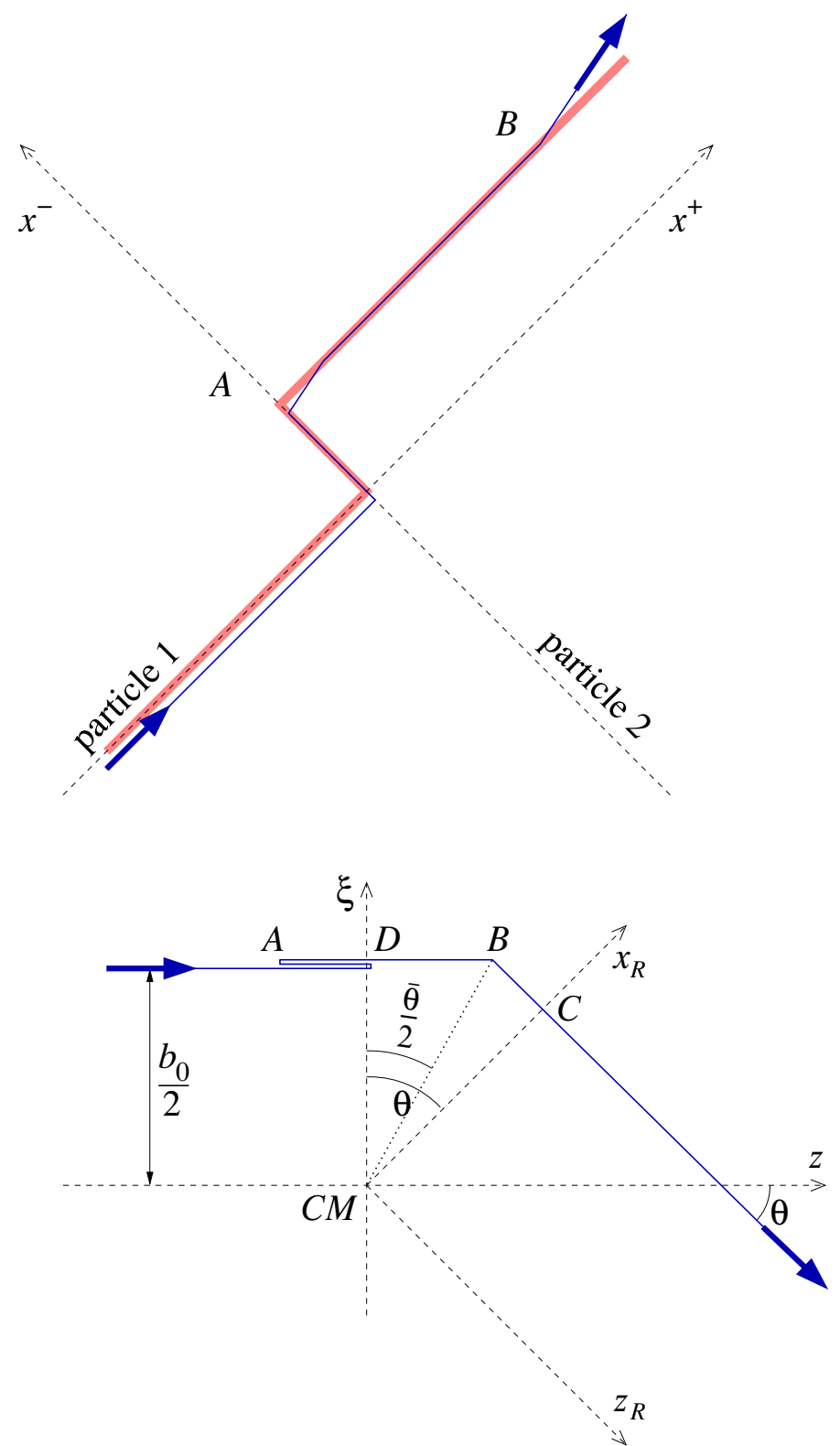

Figure 3. Space-time diagram of the double-shift picture of a test particle (solid blue) by the shock-wave of particle 2 (not shown) and then by the shifted shock wave of particle 1 (thick pink line). In the upper part, the motion in the longitudinal plane is shown. In the lower part, the coordinate $\xi$ denote the transverse direction starting from the center of mass $(\mathrm{CM})$ of the two colliding particles. At the point $A(B)$ the test particle leaves the first (second) wave front. 
where $x=b_{0} / 2+\xi=0$ is "on-shell". Since $b_{0}=b \cos (\theta / 2)$, eq. (2.46) is consistent with the action determination, at leading level:

$$
\sin \frac{\theta}{2}=\pi R\left|\frac{\mathrm{d} a(b)}{\mathrm{d} b}\right|=\frac{R}{b}, \quad \Longrightarrow \quad \tan \frac{\theta}{2}=\frac{R}{b_{0}} .
$$

Besides providing the scattering angle, the double-shift picture has a strict analogy with the wave packet motion in section 2.2. The former can be summarized in 3 steps:

a) The "come-back" motion from $z=0^{-}$back to $z=0^{+}$, taking the time $t_{D}=$ $2 \pi R \bar{a}(0)=2 \pi R a(b)$ which is just the retardation time with respect to travel at the speed of light;

b) the motion along the residual shift $\Delta z$, taking time

$$
\Delta z=\pi R\left[\tilde{a}-\bar{a}\left(\frac{b}{2}+\xi\right)\right]=\xi \tan \frac{\bar{\theta}}{2}
$$

where we have defined the angular shift $\bar{\theta}$ as in figure 3 ;

c) The motion along the deflected trajectory, taking time

$$
\xi \sin \theta-\Delta z \cos \theta=\xi \sin \left(\theta-\frac{\bar{\theta}}{2}\right) / \cos \left(\frac{\bar{\theta}}{2}\right) .
$$

By comparison, in the wave packet motion the delay time is just the same, while steps (b) (travel to point $\vec{x}_{0}=\vec{b}$ joining incoming and outgoing trajectories) and (c) (travel to $\left.z_{R}=0\right)$ both take time $\xi \tan (\theta / 2)$.

Although different, the expressions (2.48) and (2.49) correspond to the total time

$$
2 \xi \tan \frac{\theta}{2}\left[1+\sin \left(\frac{\theta}{2}\right) \sin \left(\frac{\theta-\bar{\theta}}{2}\right) / \cos \left(\frac{\bar{\theta}}{2}\right)\right]
$$

whose difference with the former is of relative order $R^{2} / b^{2}$, because both $\theta$ and $\bar{\theta}$ are of order $R / b$. Therefore the two pictures coincide at leading order.

Similarly, the outgoing impact parameter is

$$
\xi \cos \theta+\sin \theta \Delta z=\xi\left[1-2 \sin \left(\frac{\theta}{2}\right) \sin \left(\frac{\theta-\bar{\theta}}{2}\right) / \cos \left(\frac{\bar{\theta}}{2}\right)\right]
$$

which differs from the incoming one by relative order $R^{2} / b^{2}$ also. Furthermore both differences vanish in the limit $\theta=\bar{\theta}$, which is obtained by setting

$$
a\left(\frac{b_{0}}{2}+\xi\right)=\tilde{a}+a^{\prime} \xi, \quad \text { with } \tilde{a} \text { and } a^{\prime} \text { constants. }
$$

This model, that we could call the "linear model", could be described by the scattering of extended sources, in which the energy of each source inside a disk of radius $r$ increases like $r$.

We conclude that the double-shift picture provides us with some understanding of the evolution of the metric at collision and after, which is satisfactory at leading level, even if a full account of the collision process is lacking. The basic features argued so far are: 


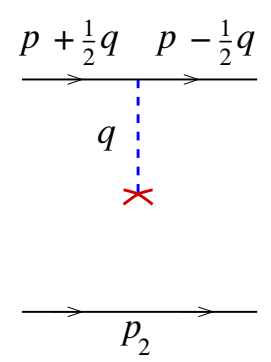

(a)

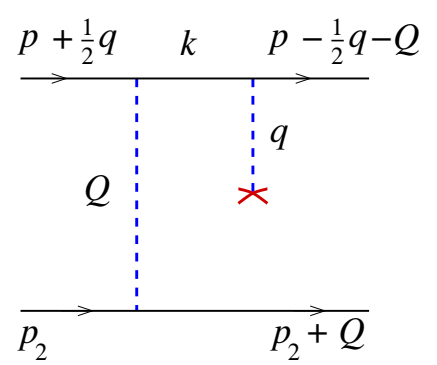

(b)

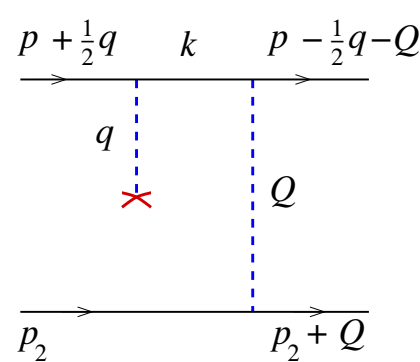

(c)

Figure 4. Simplest eikonal diagrams with one insertion of the $h_{--}$field (red cross). The parametrization of momenta is choosen so as to simplify the the calculations.

- The existence of a delay time $t_{D}=2 \pi R a(b)$ which causes a shift of either shock wave around $z=0$ in the form

$$
h_{++}=2 \pi R \bar{a}(x) \delta\left(x^{+}-2 \pi R a(b) \Theta\left(t-t_{D}\right) \Theta\left(t_{C}-t\right)\right)
$$

around particle 1, with a similar one for particle 2;

- The deflection of the particle trajectories, causing a rotation by the scattering angle $\theta$ for $t>t_{B}$ and reaching $z_{R}=0$ at the collision time $t=t_{C}$

$$
\begin{aligned}
& h_{++}=2 \pi R \bar{a}\left(\boldsymbol{x}_{R}\right) \delta\left(x_{R}^{+}-2 \pi R a(b) \Theta\left(t-t_{C}\right)\right) \\
& h_{--}=2 \pi R a\left(\boldsymbol{x}_{R}\right) \delta\left(x_{R}^{-}-2 \pi R \bar{a}(b) \Theta\left(t-t_{C}\right)\right),
\end{aligned}
$$

where the rotated coordinates are

$$
\begin{aligned}
& x_{R}^{ \pm}=t \pm z \cos \theta \\
& \boldsymbol{x}_{R}^{1}=\boldsymbol{x}^{1} \cos \theta-\boldsymbol{x}^{2} \sin \theta \\
& \boldsymbol{x}_{R}^{2}=\boldsymbol{x}^{1} \sin \theta+\boldsymbol{x}^{2} \cos \theta .
\end{aligned}
$$

Therefore, the particle motion occurs explicitly in the expression of the metric, that we could call at this point, a self-consistent leading metric.

\section{Shifted shock-wave fields from the improved eikonal model}

We have seen before how the improved eikonal model is able to describe particle motion at leading level (section 2.2), and how this feature can be incorporated by trajectory shifts in the corresponding shock-waves (section 2.3). We want now to show how the shock-wave fields can be explicitly derived from the sum of diagrams describing eikonal scattering, as defined in section 2.1. We confirm in this way the delay shifts introduced previously, leading eventually to the scattered energy-momentum.

In order to calculate the fields $h_{++}$and $h_{--}$we should consider the eikonal diagrams (figure 1) in presence of a linearly coupled external source $\left(T^{++}, T^{--}\right)$which generates the 
fields by a functional derivative of the corresponding semiclassical $S$-matrix or, in other words, of the corresponding action. We can write

$$
h_{--}(x)=\left.S^{-1} \frac{\delta S}{\delta T^{--}(x)}\right|_{T^{--}=0}
$$

and compute the result by inserting the $T^{--}$source on the eikonal diagrams, in all possible ways.

Since the external current diagrams generate insertions of momentum $q$ and thus generally do not conserve energy-momentum, we should restore time-ordered integrations in order to evaluate them properly. Since $h_{--}$is coupled to $p^{+}$only, the $p^{-}$conservation is not affected, and it is sufficient to consider $x^{+}$-ordered diagrams as depicted in figure 4. Let us first consider the small scattering angle kinematics $\theta \ll 1$, in which the longitudinal dynamics is separated from the transverse one.

In order to understand the issue, let us start from the simplest diagrams in figure 4. We notice that, while the $Q$-exchange in the eikonal line has negligible $Q^{ \pm}=\mathcal{O}\left(Q^{2} / \sqrt{s}\right)$ because of two mass-shell conditions $\left(p_{1}-Q\right)^{2}=0=\left(p_{2}+Q\right)^{2}$, the $q$-exchange has only one, $(k-q)^{2}=0$, so that some $q^{+} \gtrsim|\boldsymbol{q}|$ is still allowed, provided $q^{+} \ll \sqrt{s}$. Therefore $q^{+} q^{-}=$ $\mathcal{O}\left(\boldsymbol{q}^{2} q^{+} / \sqrt{s}\right)$ is still negligible, but the $q^{+}$leakage should be considered in the $p^{+}$evolution.

In the example of figure 4 we choose to couple the $T^{--}$current to initial and final particles of type 1 symmetrically, ${ }^{5}$ so that a $q^{+}$-independent factor of $\kappa^{2} p^{+} /\left(2 \boldsymbol{q}^{2}\right)$ is factored out. In this way, the lowest-order diagram in figure 4.a yields the Born-level field

$$
h_{--}^{(0)}\left(x^{+}, x^{-}, \boldsymbol{x}\right)=4 \pi G \sqrt{s} \int \frac{\mathrm{d}^{2} \boldsymbol{q}}{(2 \pi)^{2}} \frac{\mathrm{e}^{\mathrm{i} \boldsymbol{q} \cdot \boldsymbol{x}}}{\boldsymbol{q}^{2}} \int \frac{\mathrm{d} q^{+}}{4 \pi} \mathrm{e}^{-\mathrm{i} \frac{q^{+}}{2} x^{-}}=2 \pi R a_{0}(\boldsymbol{x}) \delta\left(x^{-}\right)
$$

where $a_{0}(\boldsymbol{x})=\frac{1}{2 \pi} \log \frac{L^{2}}{\boldsymbol{x}^{2}}$ is the leading profile function of the shock-wave.

On the other hand, the $Q$-exchange yields the leading eikonal $\mathrm{i} a_{0}(\boldsymbol{b})$ coupled to the charge $2 \pi G \bar{p}^{-}\left(p^{+}+\frac{1}{2} q^{+}\right)=2 \pi G\left(s+\sqrt{s} \frac{1}{2} q^{+}\right)$, where $\frac{1}{2} q^{+}=\mathrm{i} \partial / \partial x^{-}$according to eq. (3.2). Therefore, diagram 4 .b predicts a (subleading) contribution to $h_{--}$

$$
h_{--}^{(1 b)}=2 \pi R a_{0}(\boldsymbol{x})\left[-\pi R \delta^{\prime}\left(x^{-}\right) a_{0}(\boldsymbol{b}) \Theta\left(x^{+}\right)\right]
$$

while diagram 4.c yields

$$
h_{--}^{(1 c)}=2 \pi R a_{0}(\boldsymbol{x})\left[\pi R \delta^{\prime}\left(x^{-}\right) a_{0}(\boldsymbol{b}) \Theta\left(-x^{+}\right)\right]
$$

because of the opposite sign in $q^{+}$and $x^{+}$.

We shall interpret the results (3.3) as the first order expansion in $R / x^{-}$of a shifted field

$$
h_{--}\left(x^{+}, x^{-}, \boldsymbol{x}\right)=2 \pi R a_{0}(\boldsymbol{x}) \delta\left(x^{-}-2 \pi R \frac{1}{2} \epsilon\left(x^{+}\right) a_{0}(\boldsymbol{b})\right)
$$

which is retarded (advanced) for $x^{+}>0\left(x^{+}<0\right)$. Therefore, the result (3.4) confirms the delay-shift of section 2.3, in a form which is appropriate for the calculation of the real part of the action $\Re A$, or to the principal-value prescription of the $x^{-}, x^{+}$propagator. 


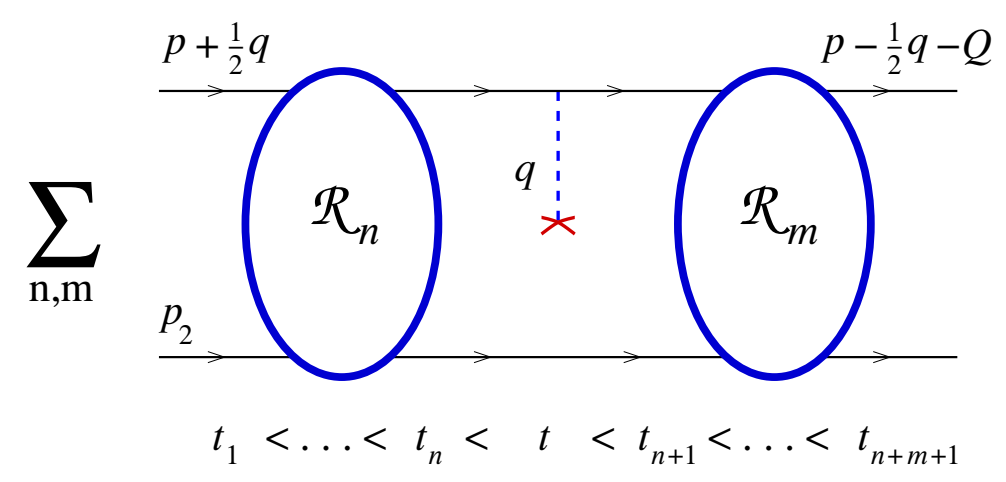

Figure 5. Higher order eikonal diagrams with one insertion of the field $h_{--}$(red cross). The blobs represent ladders of eikonal exchanges.

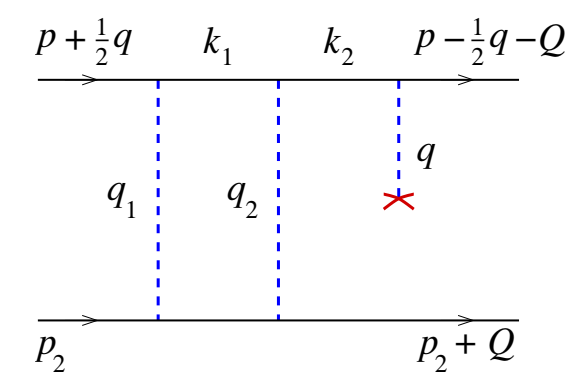

Figure 6. Eikonal diagram with two graviton exchanges and one insertion of the field $h_{--}$(red cross).

The higher-order contributions to the shifted field are computed from their definition in figure 5. In order to do the explicit calculation, we have to explain in more detail how the $x^{+}$-ordering just mentioned is generated from the usual $t$-ordering in perturbation theory. We shall work off energy-shell, but on mass-shell for the intermediate particles so that, in the example of figure $6, E_{0}=|\vec{p}|=\left|\vec{k}_{0}\right|, E_{1}=\left|\vec{k}_{1}\right|, \ldots, \bar{E}_{0}=\left|\vec{k}_{0}\right|$. Therefore, to each eikonal exchange, we assign the energy differences in the center of mass frame $\Delta_{i}=\Delta\left(\boldsymbol{k}_{i}, \boldsymbol{k}_{i-1}\right)$, e.g.,

$$
\begin{aligned}
& \Delta_{1} \equiv\left|\vec{k}_{1}\right|+\left|\vec{k}_{1}\right|-\left|\vec{k}_{0}\right|-\left|\vec{k}_{0}\right|=2\left(\left|\vec{p}-\vec{q}_{1}\right|-|\vec{p}|\right) \simeq 2 \frac{\vec{q}_{1}^{2}-2 \vec{p} \cdot \vec{q}_{1}}{2|\vec{p}|} \\
& \Delta_{2}=2\left(\left|\vec{k}_{1}-\vec{q}_{2}\right|-\left|\vec{k}_{1}\right|\right) \simeq 2 \frac{\vec{q}_{2}^{2}-2 \vec{k}_{1} \cdot \vec{q}_{2}}{2\left|\vec{k}_{1}\right|}=2 \frac{\vec{Q}^{2}-2 \vec{p} \cdot \vec{Q}}{2|\vec{p}|}
\end{aligned}
$$

and so on. ${ }^{6}$ The ordered time integrations, up to time $t$ of the field, yield therefore the factor

$$
\frac{\exp \left[\mathrm{i}\left(\Delta_{1}+\Delta_{2}\right) t\right]}{\left[\varepsilon+\mathrm{i}\left(\Delta_{1}+\Delta_{2}\right)\right]\left[\varepsilon+\mathrm{i} \Delta_{1}\right]} \rightarrow \frac{\exp \left[\mathrm{i}\left(\Delta_{1}+\Delta_{2}\right) t\right]}{2\left(\varepsilon+\mathrm{i} \Delta_{2}\right)\left(\varepsilon+\mathrm{i} \Delta_{1}\right)}
$$

\footnotetext{
${ }^{5}$ Furthermore, we shall weight the initial and final states in (3.1) with a wave function having energy centered around $p^{+}=\sqrt{s}=p_{2}^{-}$, which amounts to setting $p_{1}^{+}=\sqrt{s}+\frac{1}{2} q^{+}$and $p_{1}^{+}=\sqrt{s}-\frac{1}{2} q^{+}$.

${ }^{6}$ The approximate evaluation of the $\Delta_{i}$ 's occurring in the last equation's line is sufficient to our purposes, because we shall need it around $\Delta_{i}=0$. The argument is therefore valid for the finite-angle kinematics too.
} 


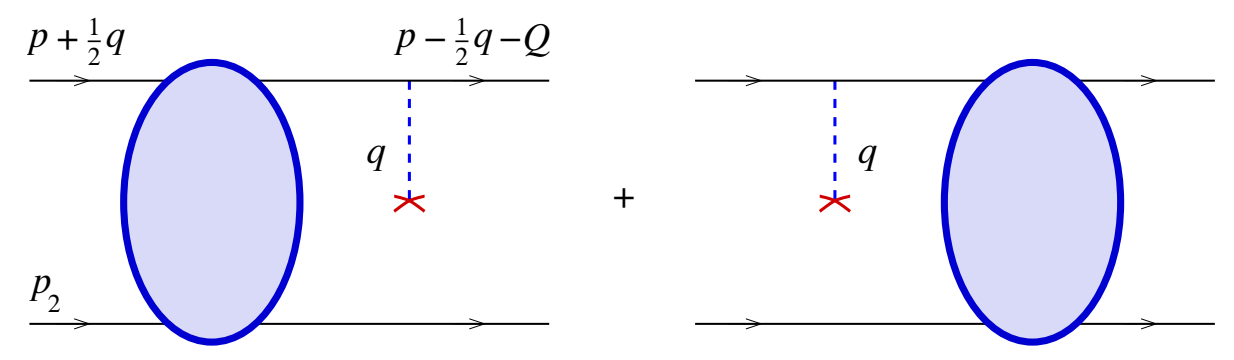

Figure 7. Structure of the eikonal diagrams contributing to the field $h_{--}$.

where the last expression is obtained after $1 \leftrightarrow 2$ symmetrization, because of the integrations on $\Delta_{1}, \Delta_{2}$ induced by $\mathrm{d}^{3} q_{1}, \mathrm{~d}^{3} q_{2}$, with a factor which is symmetrical under $\vec{q}_{i}$ permutations.

Finally the factorized $\Delta_{i}$ integrations induced by the angular ones on the $\vec{q}_{i}$ 's are done separately, by using the identity

$$
\frac{1}{2 \pi} \int \mathrm{d} \Delta \frac{\mathrm{e}^{\mathrm{i} \Delta t}}{\varepsilon+\mathrm{i} \Delta}=\Theta(t)+\mathcal{O}\left(\mathrm{e}^{-\sqrt{s}|t|}\right)
$$

in which the integration contour is closed in the upper (lower) half-plane for $t>0(t<0)$ by noting that corrections due to the large $\Delta$ contour are exponentially small in $\left|\Delta_{\max }\right|=$ $\mathcal{O}\left(\left|\vec{q}_{i}\right|\right)=\mathcal{O}(\sqrt{s})$ in our finite angle kinematics. Similarly, the time and $\Delta$ integrations can be done after the insertion, and yield

$$
\frac{1}{2 \pi} \int \mathrm{d} \Delta \frac{\mathrm{e}^{\mathrm{i} \Delta t}}{\varepsilon-\mathrm{i} \Delta} \simeq \Theta(-t)
$$

This means that eikonal echanges occurring both before and after the insertion are suppressed, ${ }^{7}$ because of $\Theta(t) \Theta(-t)=0$, so that the whole sum reduces to the diagrams in figure 7 .

In order to compute them we first diagonalize the scattering amplitude in impact parameter $\boldsymbol{b}$ space and we then compute the inserted field by Fourier transform in the $q^{\mu}$ variables, in order to yield its $x^{-}, x^{+}, \boldsymbol{x}$ dependence. The non-trivial point about impact parameter transform is that it is 2-dimensional, while the integration variables $\vec{q}_{1}$ and $\vec{Q}-\vec{q}_{1}$ (in, say figure 6) are 3-dimensional in the finite angle kinematics. Fortunately, the conditions $\Delta_{1}=0=\Delta_{2}$ fix the 3 -components of both $\vec{q}_{1}$ and $\vec{Q}$, by determining the directions of the respective Breit frames. We thus have the Bessel transform

$$
\frac{1}{2} \int \frac{\mathrm{d}^{3} q_{1}}{2 \pi^{2} q_{1}^{2}} \delta\left(q_{1} \cos \alpha_{1}-\frac{q_{1}^{2}}{\sqrt{s}}\right) \frac{\mathrm{d}^{3} Q}{2 \pi^{2}} \frac{\delta\left(Q \cos \beta_{1}-\frac{Q^{2}}{\sqrt{s}}\right) J_{0}(b Q)}{Q^{2}+q_{1}^{2}-2 Q q_{1}\left(\cos \alpha_{1} \cos \beta_{1}-\sin \alpha_{1} \sin \beta_{1} \cos \phi_{1}\right)}
$$

\footnotetext{
${ }^{7}$ Of course, a more refined calculation may introduce a spread $\Delta t \sim 1 / \sqrt{s}$ in the $\Theta$-functions so that overlap of subsequent contributions is allowed for a limited time. The uncertainty so introduced in the time of shift is expected to be larger than $1 / \sqrt{ } s$ because of the number of coherent reinteractions, but in any case smaller than $R$, which would correspond to the maximal number $G s / \hbar$ of leading eikonal scatterings.
} 
which, by the clever identity (2.7) yields the same result as the 2-dimensional azimuthal average

$$
\begin{aligned}
& \frac{\mathrm{i}^{2}}{2} \int \frac{q_{1} \mathrm{~d} q_{1} \mathrm{~d} \phi_{1}}{2 \pi^{2} q_{1}^{2}} \frac{Q \mathrm{~d} Q \mathrm{~d} \phi_{Q}}{2 \pi^{2}\left(q_{1}^{2}+Q^{2}-2 Q q_{1} \cos \phi_{1}\right)} \mathrm{e}^{-\mathrm{i} b Q \cos \phi_{Q}} \\
& =\frac{\mathrm{i}^{2}}{2} \int \frac{\mathrm{d}^{2} \boldsymbol{q}_{1} \mathrm{~d}^{2} \boldsymbol{Q}}{\left(2 \pi^{2}\right)^{2}\left(\boldsymbol{Q}-\boldsymbol{q}_{1}\right)^{2} \boldsymbol{q}_{1}^{2}} \mathrm{e}^{-\mathrm{i} \boldsymbol{b} \cdot\left(\boldsymbol{Q}-\boldsymbol{q}_{1}+\boldsymbol{q}_{1}\right)}=\frac{1}{2}\left(a_{0}(\boldsymbol{b})\right)^{2}
\end{aligned}
$$

which is a 2-dimensional convolution diagonalized in $\boldsymbol{b}$-space.

We thus obtain that the amplitude in front of the field insertion resums to the exponentiated result

$$
\sum_{n} \frac{1}{n !}\left(\mathrm{i} 2 \pi G s a_{0}(b)\right)^{n}=\mathrm{e}^{\mathrm{i} 2 \pi G s a_{0}(b)}=S(b, s)
$$

and cancels out with the factor $S^{-1}$ in eq. (3.1).

We are left with the calculation of the $h_{--}$field itself. Notice that in figure 7.b the insertion is on the incoming leg $\vec{p}$, while in figure 7.a it occurs on the final leg $\vec{k}=\vec{p}-\vec{Q}$. Since $|\vec{p}-\vec{Q}|=|\vec{p}|$ because of the mass-shell plus energy-conservation constraints, the difference is just a rotation $\vec{k}=\vec{p}_{R}$ by the angle $\sin \frac{\theta}{2}=Q / \sqrt{s}$. When inserted in the field propagator it leads to a rotated variable ${ }^{8} \vec{x}_{R}$ with a mass-shell condition ${ }^{9}(p-q)^{2}=$ $-q^{-}\left(\sqrt{s}-q^{+}\right)-\boldsymbol{q}^{2}=0$, and we get

$$
\int \frac{\mathrm{d}^{4} q}{(2 \pi)^{3}} \frac{\mathrm{e}^{\mathrm{i} \vec{q} \cdot \vec{x}_{R}} \mathrm{e}^{-\mathrm{i} q_{0} t}\left(2 p^{0}-q^{0}\right)}{\vec{q}^{2}-q_{0}^{2}} \delta\left((q-p)^{2}\right)=\int \frac{\mathrm{d}^{2} \boldsymbol{q}}{(2 \pi)^{2}} \frac{\mathrm{e}^{\mathrm{i} \boldsymbol{q} \cdot \boldsymbol{x}_{R}} \mathrm{e}^{-\mathrm{i} \frac{q^{+}}{2} x_{R}^{-}}}{\boldsymbol{q}^{2}}\left(p^{+}-\frac{1}{2} q^{+}\right) \frac{\mathrm{d} q^{+}}{4 \pi},
$$

where we have neglected the exponent dependence on $q^{-} x^{+}$, when $q^{-}=-\boldsymbol{q}^{2} /\left(\sqrt{s}-q^{+}\right)$is a very small variable if we make the natural assumption $|\vec{q}| \ll \sqrt{s}$, insuring that the field insertion does not modify the scattering angle.

Finally, by taking the value $p^{+}=\sqrt{s}+\frac{1}{2} q^{+}$of the initial energy as we did in the beginning, we get for the field a factorized value proportional to $\delta\left(x^{-}\right)$which translates $\Theta(t)$ into $\Theta\left(x^{+}\right)$. The complete resummation in figure 5 is thus summarized in the exponentiated form

$$
\begin{aligned}
h_{--}(x)= & S^{-1} \exp \left[2 \pi G \sqrt{s} a_{0}(b)\left(\mathrm{i} \sqrt{s}-\frac{\partial}{\partial x^{-}}\right) \Theta\left(x^{+}\right)+\left(\mathrm{i} \sqrt{s}+\frac{\partial}{\partial x^{-}}\right) \Theta\left(-x^{+}\right)\right] \times \\
& \times\left\{2 \pi R\left[\Theta\left(-x^{+}\right) \delta\left(x^{-}\right) a_{0}(\boldsymbol{x})+\Theta\left(x^{+}\right) \delta\left(x_{R}^{-}\right) a_{0}\left(\boldsymbol{x}_{R}\right)\right]\right\} \\
= & 2 \pi R\left[\delta\left(x_{R}^{-}-\pi R a_{0}(b)\right) a_{0}\left(\boldsymbol{x}_{R}\right) \Theta\left(x^{+}\right)+\delta\left(x^{-}+\pi R a_{0}(b)\right) a_{0}(\boldsymbol{x}) \Theta\left(-x^{+}\right)\right] .
\end{aligned}
$$

An identical calculation with the exchange $(+\leftrightarrow-)$ yields an analogous result for the field $h_{++}(x)$. The overall situation is depicted in figure 8 , where we show the shifted (and slightly rotated) shock waves, on which the source particles lie before and after the

\footnotetext{
${ }^{8}$ The ++ projection should be rotated also with the $z$-axis in the direction of the deflected longitudinal direction.

${ }^{9}$ Strictly speaking, we should replace this mass-shell condition by the denominator $\left[(p-q)^{2}+\mathrm{i} \varepsilon\right]^{-1}$. However, for $x^{+}>0$ the contour can be closed on the pole, with the same result, while for $x^{+}<0$ it vanishes. This remark confirms the ordering $\Theta\left(x^{+}\right)$(see appendix A).
} 


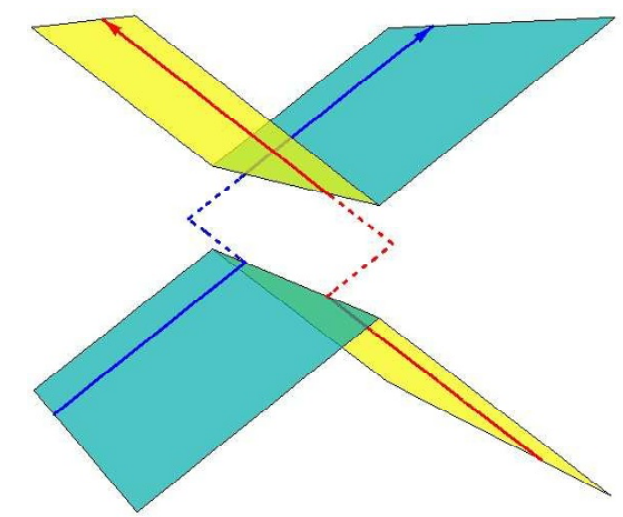

Figure 8. Graphical representation of the shifted shock-waves in Minkowski space (only one transverse dimension is visible). The cyan (yellow) half-planes show the support of the field $h_{--}$ $\left(h_{++}\right)$generated by particle $1(2)$, here represented by the blue (red) line.

collision (solid lines). The evolution of the particles during the collision actually depends on the reference frame used to describe their motion. For instance, a time-like observer that crosses first shock 2 (the one on the bottom-right) describes the scattering in two steps: firstly the shock 2 drags shock 1 and particle 1 by an amount $2 \pi R \bar{a}(x)$ in the $x^{-}$direction; then shock 1 drags shock 2 and particle 2 in the $x^{+}$direction. In this frame particle 1 travels along the dashed blue line in figure 8. A symmetric description is given by an observer crossing first shock 1, which "sees" particle 2 moving along the dashed red line.

\section{Rescattering corrections to the reduced-action model}

\subsection{Semiclassical field equations}

The transplanckian field equations in the ACV proposal [6] were based on two main groups of results. Firstly, the ACV investigation of string-gravity showed that at transplanckian energies $G s \gg 1(R \equiv 2 G \sqrt{s} \gg 1 / \sqrt{s})$ the eikonal representation - with an eikonal operator which is calculable in principle by expanding in $\lambda_{s} / b$ and $R / b$ - yields a good representation of the scattering amplitude, and incorporates both string- and strong-gravity effects.

Secondly, in the regime $b \gtrsim R \gg \lambda_{s}$ in which string effects are supposed to be small, the irreducible eikonal diagrams are much in correspondence with the effective action of Lipatov and co-workers [17, 18], who calculated a Regge-graviton emission vertex [19] which is the building block of the effective lagrangian used in [7]. Finally, the equations of motion of the latter — by neglecting rescattering terms - were shown to yield a shock-wave solution for the fields which is the basis for the reduced-action model investigated in detail in later years [8].

Here, we have considered so far only the leading graviton-exchange kernel in the eikonal, and we have improved the amplitude representation based on it, so as to include a motion of the Breit-frame on which the exchange is defined. Next, we want to reconsider the field equations in 4-dimensions - including rescattering - and we shall provide a solution for 
the fields which fits very well in our understanding of scattering developed so far, by adding corrections which are of relative order $R^{2} / b^{2}$ and higher.

In the effective action framework, the elastic $S$-matrix of the tree diagrams in figure 9 is given in terms of the classical solutions of the lagrangian equation of motion as

$$
\begin{aligned}
S(b, s) & =\exp \left\{\frac{\mathrm{i}}{\hbar} A\left(h_{\mathrm{cl}}^{\mu \nu}\right)\right\} \\
A\left(\tilde{h}^{++}, \tilde{h}^{--}, \Phi\right) & =\int \mathrm{d}^{4} x\left(\mathcal{L}_{0}+\mathcal{L}_{e}+\mathcal{L}_{r}+T_{++} \tilde{h}^{++}+T_{--} \tilde{h}^{--}\right),
\end{aligned}
$$

where $\tilde{h}^{++} \equiv(2 / \kappa) h_{--}$and $\tilde{h}^{--} \equiv(2 / \kappa) h_{++}$are just rescaled versions of the longitudinal fields considered so far, $\Phi$ is related to the transverse field which is proportional to $h=\nabla^{2} \Phi$, and

$$
T_{--}=\kappa E \delta\left(x^{-}\right) \delta^{2}(\boldsymbol{x}), \quad T_{++}=\kappa E \delta\left(x^{+}\right) \delta^{2}(\boldsymbol{x}-\boldsymbol{b})
$$

represents (up to an unconventional but convenient factor of $\kappa$ ) the energy-momentum tensor of the colliding particles. The usual metric components are given by [8]

$$
\begin{aligned}
h_{\mu \nu} \mathrm{d} x^{\mu} \mathrm{d} x^{\nu} & \equiv \mathrm{d} s^{2}-\eta_{\mu \nu} \mathrm{d} x^{\mu} \mathrm{d} x^{\nu} \\
& =\frac{\kappa}{2}\left[\tilde{h}^{++}\left(\mathrm{d} x^{-}\right)^{2}+\tilde{h}^{--}\left(\mathrm{d} x^{+}\right)^{2}\right]+\frac{\kappa}{4}\left[\epsilon_{\mu \nu}^{T T} \nabla^{2} \Re \Phi-\epsilon_{\mu \nu}^{L T} \nabla^{2} \Im \Phi\right] \mathrm{d} x^{\mu} \mathrm{d} x^{\nu},
\end{aligned}
$$

where $\nabla^{2}$ denotes the transverse laplacian and the $\epsilon$ 's denote graviton polarizations to be specified below.

The lagrangian is conveniently written in terms of real light-cone variables $x^{ \pm}, \partial_{ \pm} \equiv$ $\partial / \partial x^{ \pm}$and a complex transverse variable $z \equiv x^{1}+\mathrm{i} x^{2}, \partial \equiv \partial / \partial z=\frac{1}{2}\left(\partial_{1}-\mathrm{i} \partial_{2}\right)$. It consists of a kinetic term

$$
\mathcal{L}_{0}=-\partial^{*} \tilde{h}^{++} \partial \tilde{h}^{--}+4 \partial_{+} \partial^{* 2} \Phi \partial_{-} \partial^{2} \Phi^{*},
$$

where the longitudinal fields have a mostly transverse propagator and the (complex) $\Phi$ field a mostly longitudinal one, of a graviton emission term

$$
\mathcal{L}_{e}=\kappa\left(\mathcal{J}|\partial|^{2} \Phi^{*}+\mathcal{J}^{*}|\partial|^{2} \Phi\right), \quad|\partial|^{2} \mathcal{J}=\partial^{* 2} \tilde{h}^{++} \partial^{2} \tilde{h}^{--}-|\partial|^{2} \tilde{h}^{++}|\partial|^{2} \tilde{h}^{--}
$$

incorporating Lipatov's vertex [19], and finally, of a rescattering term

$$
\mathcal{L}_{r}=\kappa\left(\tilde{h}^{++} \partial^{* 2} \Phi^{*} \partial_{+}^{2} \partial^{2} \Phi+\tilde{h}^{--} \partial^{2} \Phi^{*} \partial_{-}^{2} \partial^{* 2} \Phi\right) \quad+\quad\left(\partial_{+} \leftrightarrow \partial_{-}\right),
$$

which is supposed to take into account the rescattering diagrams of figure 9.c,d. This term is quadratic in $\Phi$, and is likely to play a role when the latter is large.

Notice in particular in eq. (4.6) the current $\mathcal{J}$, which describes transverse graviton emission [17] in the so-called H-diagram [6] and is non-local because of the inverse laplacian needed to find it. For this reason it has been convenient to introduce the complex field $\Phi$, which corresponds to two graviton polarizations. The detailed computation shows that

$$
h_{z \bar{z}}=\kappa|\partial|^{2} \Phi, \quad h_{z z}=\kappa \partial^{2} \Phi, \quad h_{\bar{z} \bar{z}}=\kappa \partial^{* 2} \Phi
$$




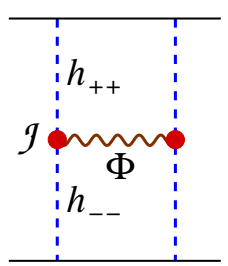

(a)

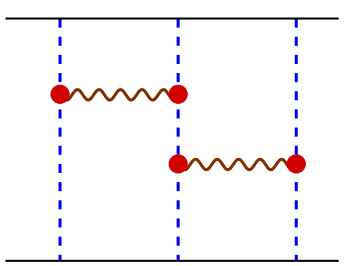

(b)

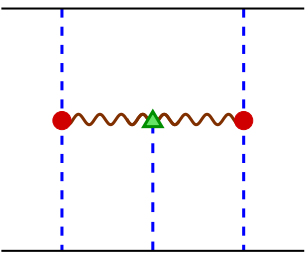

(c)

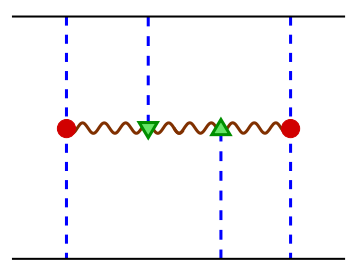

(d)

Figure 9. Diagrams providing subleading contributions to the eikonal approximation: $(a)$ the "H-diagram" representing the first correction of (relative) order $(R / b)^{2} ;(b)$ a "multi-H" diagram of order $(R / b)^{4}$ involving only Lipatov's vertices (red disks); $(c)$ the first diagram with a rescattering vertex (green triangle) is of order $(R / b)^{3}$, but actually vanishes on-shell; $(d)$ the first nonvanishing rescattering diagram is of order $(R / b)^{4}$.

and, more generally, using a notation with real-valued indices, that

$$
h_{\mu \nu}=\kappa \Re\left(-\eta_{\mu}^{*} \eta_{\nu}^{*}|\partial|^{2} \Phi\right)
$$

where

$$
\eta^{\mu}(k) \equiv \varepsilon_{L}^{\mu}+\mathrm{i} \varepsilon_{T}^{\mu}=\left(\frac{k^{3}}{|\boldsymbol{k}|}, \mathrm{i} \varepsilon, \frac{k^{0}}{|\boldsymbol{k}|}\right)
$$

so that we have the polarizations

$$
\varepsilon_{T T}^{\mu \nu}=\left(\varepsilon_{T}^{\mu} \varepsilon_{T}^{\nu}-\varepsilon_{L}^{\mu} \varepsilon_{L}^{\nu}\right), \quad \varepsilon_{L T}^{\mu \nu}=\left(\varepsilon_{L}^{\mu} \varepsilon_{T}^{\nu}+\varepsilon_{T}^{\mu} \varepsilon_{L}^{\nu}\right), \quad \varepsilon_{\mu \nu}^{i} \varepsilon_{j}^{\mu \nu}=2 \delta_{j}^{i} \quad(i, j=T T, L T) .
$$

The resulting equations of motion are given by

$$
\begin{gathered}
|\partial|^{2} \tilde{h}^{++}+\kappa\left[\partial^{2}\left(\Phi^{*} \partial^{* 2} \tilde{h}^{++}+\partial^{* 2}\left(\Phi \partial^{2} \tilde{h}^{++}\right)-|\partial|^{2}\left(\left(\Phi+\Phi^{*}\right)|\partial|^{2} \tilde{h}^{++}\right)\right]+\frac{1}{2} \kappa \sqrt{s} \delta^{2}(\boldsymbol{x}) \delta\left(x^{-}\right)\right. \\
=-\kappa\left(\partial^{2} \Phi^{*} \partial_{-}^{2} \partial^{* 2} \Phi+\left(\partial \leftrightarrow \partial^{*}\right)\right) \\
4 \partial_{+} \partial_{-}|\partial|^{4} \Phi-\kappa\left[\partial^{* 2} \tilde{h}^{++} \partial^{2} \tilde{h}^{--}-|\partial|^{2} \tilde{h}^{++}|\partial|^{2} \tilde{h}^{--}\right] \\
=\kappa\left[\partial^{* 2}\left(\tilde{h}^{++} \partial_{+}^{2} \partial^{2} \Phi\right)+\partial^{2}\left(\tilde{h}^{--} \partial_{-}^{2} \partial^{* 2} \Phi\right)+\left(\partial \leftrightarrow \partial^{*}\right)\right],
\end{gathered}
$$

where the rescattering terms are set on the r.h.s., with similar equations for $\tilde{h}^{--}$and $\Phi^{*}$.

Notice that in eqs. (4.12) the impinging particles are represented by the frozen external sources $T^{++}$and $T^{--}$written in eq. (4.3), which pertain to the collinear kinematics used before. According to this small-angle approach, scattering comes out because of a nontrivial action, but is not taken into account in the energy-momentum of the particles. Shortly, we shall take a somewhat different attitude.

\subsection{The reduced-action without rescattering}

We start recalling [7] that, if the rescattering terms are dropped, eqs. (4.12) admit a shockwave solution of the form

$$
4 \tilde{h}_{--}=\tilde{h}^{++}=\kappa \sqrt{s} \delta\left(x^{-}\right) a(\boldsymbol{x})
$$




$$
\begin{aligned}
4 \tilde{h}_{++}=\tilde{h}^{--} & =\kappa \sqrt{s} \delta\left(x^{+}\right) \bar{a}(\boldsymbol{x}) \\
\Phi & =\frac{\kappa^{3} s}{2} \frac{1}{2} \Theta\left(x^{+} x^{-}\right) \phi(\boldsymbol{x}),
\end{aligned}
$$

where the metric component $h_{--}=(\kappa / 2) \tilde{h}^{++}=2 \pi R a(\boldsymbol{x}) \delta\left(x^{-}\right)$are of AS type [11] while the transverse field $\Phi$ has support inside the whole light-wedges $x^{+} x^{-} \geq 0$. The latter propagation, of retarded plus advanced type, corresponds to the principal value part of the Feynman propagators, as is appropriate for the real part of the amplitude.

The field equations (4.12) induce a set of differential equations on $a, \bar{a}$ and $\phi$, which can be solved on the basis of proper boundary conditions which make them regular, i.e., ultraviolet safe. Furthermore, by replacing the expressions (4.13) into (4.12) and omitting the rescattering term, we obtain the ACV equations of motion and the corresponding reduced action

$$
\begin{aligned}
A_{R}=2 \pi G s\left\{a(\boldsymbol{b})+\bar{a}(0)+\int \mathrm{d}^{2} \boldsymbol{x}\right. & {\left[-\frac{1}{2} \boldsymbol{\nabla} a \cdot \nabla \bar{a}-\frac{1}{2}(\pi R)^{2}\left|\nabla^{2} \phi\right|^{2}\right.} \\
+ & \left.\left.(2 \pi R)^{2}\left(\phi^{*}\left(\partial^{* 2} a \partial^{2} \bar{a}-|\partial|^{2} a|\partial|^{2} \bar{a}\right)+\text { h.c. }\right)\right]\right\},
\end{aligned}
$$

where we have freely performed some integration by parts, assuming a smooth enough ultraviolet behaviour of the solutions, and we have replaced the current $\mathcal{J}$ by its reduced counterpart $\mathcal{H}$ :

$$
\mathcal{J} \equiv \frac{\kappa^{2} s}{2} \delta\left(x^{-}\right) \delta\left(x^{+}\right) \mathcal{H} \quad \Longrightarrow \quad \mathcal{H}=\frac{2}{|\partial|^{2}}\left(\partial^{* 2} a \partial^{2} \bar{a}-|\partial|^{2} a|\partial|^{2} \bar{a}\right) .
$$

We state below some of the properties of the RAM solutions [8] in the particularly simple case of axisymmetric sources like particle-ring scattering - which is relevant for the azimuthal averaged particle-particle case.

By assuming the real fields $a\left(r^{2}\right), \bar{a}\left(r^{2}\right)$ and the complex field $\phi\left(r^{2}\right)$ to be functions of $r^{2} \equiv|\boldsymbol{x}|^{2}$ only, and by calling $s\left(r^{2}\right), \bar{s}\left(r^{2}\right)$ the axisymmetric sources, we soon realize that the action $A_{R}$ can be recast in one-dimensional form. This is because the current $\mathcal{H}$ defined by eq. (4.15) is simply obtained in this case by setting $\dot{\mathcal{H}}=-2 \dot{a} \dot{\bar{a}}$ - where the dot denotes the $r^{2}$-derivative - and is therefore real and axisymmetric also. As a consequence, the interaction term involves $\Re \phi$ only and is proportional to the $a \bar{a}$ kinetic term as follows

$$
A_{R}=2 \pi^{2} G s \int \mathrm{d} r^{2}\left[\bar{s} a+s \bar{a}-2 \rho \dot{a} \dot{\bar{a}}-\frac{2}{(2 \pi R)^{2}}(1-\dot{\rho})^{2}\right] \quad\left(\cdot \equiv \frac{\mathrm{d}}{\mathrm{d} r^{2}}\right)
$$

Here we have replaced $\phi$ by the auxiliary field $\rho\left(r^{2}\right)$ - a sort of renormalized squared distance - defined by

$$
\rho \equiv r^{2}\left[1-(2 \pi R)^{2} \dot{\phi}\right], \quad h \equiv \nabla^{2} \phi=4 \frac{\mathrm{d}}{\mathrm{d} r^{2}}\left(r^{2} \dot{\phi}\right)=\frac{1}{(\pi R)^{2}}(1-\dot{\rho})
$$

which incorporates the $\phi-a-\bar{a}$ interaction. Furthermore, the field $\phi$ is now taken to be realvalued, describing the $T T$ polarization only. The external axisymmetric sources $s$ and $\bar{s}$ 
are able to approximately describe the particle-particle case by setting $s\left(r^{2}\right)=\delta\left(r^{2}\right) / \pi$, $\bar{s}\left(r^{2}\right)=\delta\left(r^{2}-b^{2}\right) / \pi$, where the azimuthal ACV averaging procedure is assumed.

The equations of motion, derived from the reduced-action (4.16) and specialized to the case of particles at impact parameter $b$ (with the axisymmetric sources just quoted), provide the profile functions

$$
\dot{a}=-\frac{1}{2 \pi \rho}, \quad \dot{\bar{a}}=-\frac{1}{2 \pi \rho} \Theta\left(r^{2}-b^{2}\right),
$$

from the analogue of eq. (4.12a), and the $\rho$-field (or $\phi$-field)

$$
\ddot{\rho}=\frac{1}{2 \rho^{2}} \Theta\left(r^{2}-b^{2}\right), \quad \dot{\rho}^{2}+\frac{1}{\rho}=1 \quad(r>b)
$$

from the analogue of eq. (4.12b). Eq. (4.19) shows a "Coulomb" potential in $\rho$-space, which is repulsive for $\rho>0$, acts for $r>b$ and plays an important role in the tunneling phenomenon [14]. By replacing the equation of motion (4.18) into eq. (4.16), the reduced action can be expressed in terms of the $\rho$ field only, and takes the simple form

$$
A_{R}=-G s \int \mathrm{d} r^{2}\left[\frac{1}{R^{2}}(1-\dot{\rho})^{2}-\frac{1}{\rho} \Theta\left(r^{2}-b^{2}\right)\right] \equiv-\int_{0}^{\infty} \mathrm{d} r^{2} \mathcal{L}\left(\rho, \dot{\rho}, r^{2}\right)
$$

which is the one we shall consider at quantum level in the following.

The effective metric generated by the axisymmetric fields $\rho, a$ and $\bar{a}$ was calculated [8] on the basis of the complete form of the shock-wave (4.13) and is given by

$$
\begin{aligned}
\mathrm{d} s^{2}= & -\mathrm{d} x^{+} \mathrm{d} x^{-}\left[1-\frac{1}{2} \Theta\left(x^{+} x^{-}\right)(1-\dot{\rho})\right] \\
& +\left(\mathrm{d} x^{+}\right)^{2} \delta\left(x^{+}\right)\left[2 \pi R \bar{a}\left(r^{2}\right)-\frac{1}{4}(1-\dot{\rho})\left|x^{-}\right|\right] \\
& +\left(\mathrm{d} x^{-}\right)^{2} \delta\left(x^{-}\right)\left[2 \pi R a\left(r^{2}\right)-\frac{1}{4}(1-\dot{\rho})\left|x^{+}\right|\right] \\
& +\mathrm{d} r^{2}\left[1+2(\pi R)^{2} \Theta\left(x^{+} x^{-}\right) \dot{\phi}\right]+\mathrm{d} \theta^{2} r^{2}\left[1+2(\pi R)^{2} \Theta\left(x^{+} x^{-}\right)\left(\dot{\phi}+2 r^{2} \ddot{\phi}\right)\right] .
\end{aligned}
$$

This metric is dynamically generated and may be regular or singular at short distances, depending on the behaviour of the field solutions themselves. It is not fully consistent, however, since it does not take into account the longitudinal shifts that the fields cause to each other. In the following sections we shall compute such shifts at subleading level and we shall present the improved expression of the self-consistent metric.

\subsection{The H-diagram: scattering angle and shifts}

The first nontrivial use of the reduced action (4.14) is the calculation of the first order correction to the Einstein deflection [6] which is due to the H-diagram contribution to the action (figure 9.a). The latter is obtained by expanding the action (4.14) and the corresponding equations of motion (quoted in eqs. (4.18), (4.19) for the axisymmetric case) in the parameter $R^{2} / b^{2}$ where $R \equiv 2 G \sqrt{s}$ is the gravitational radius and $b$ is the impact 
parameter conjugated to the transverse momentum $Q$ (and related to the true impact parameter $b_{0}$ by eq. (2.29)).

By expanding the equations of motion we obtain first order corrections to the profile function

$$
a(\boldsymbol{x})=a_{0}(\boldsymbol{x})+a_{1}(\boldsymbol{x})+\cdots
$$

and to the transverse field

$$
h(\boldsymbol{x})=\nabla^{2} \phi(\boldsymbol{x})=h_{0}(\boldsymbol{x})+\cdots=\frac{2}{|\partial|^{2}}\left(\partial^{* 2} a_{0} \partial^{2} \bar{a}_{0}-|\partial|^{2} a_{0}|\partial|^{2} \bar{a}_{0}\right)+\cdots
$$

as consequence of the Lipatov's vertex $\mathcal{J}$ in eq. (4.6). It is then straighforward to obtain for the action $[8]$

$$
\begin{aligned}
a(b) & =a_{0}(b)+2 a_{H}(b)+\cdots \\
A_{R} & =2 \pi G s\left[a_{0}(b)+a_{H}(b)+\cdots\right],
\end{aligned}
$$

where

$$
a_{0}(\boldsymbol{x})=\frac{1}{2 \pi} \log \frac{L^{2}}{|\boldsymbol{x}|^{2}}, \quad a_{1}(b)=2 a_{H}(b)
$$

and the H-diagram contribution is

$$
a_{H}(b)=\frac{1}{2}(\pi R)^{2} \int \mathrm{d}^{2} \boldsymbol{x}\left|h_{0}(\boldsymbol{x})\right|^{2} .
$$

In the following, we specialize the expression (4.27) to the axisymmetric case $\mathrm{e}^{10}$ in which, according to eq. (4.17),

$$
h\left(r^{2}\right)=\frac{1}{(\pi R)^{2}}\left(1-\dot{\rho}\left(r^{2}\right)\right)
$$

and — by the equations of motion (4.19) — we obtain the result

$$
a_{H}=\frac{1}{2 \pi R^{2}} \int_{0}^{\infty} \mathrm{d} r^{2}(1-\dot{\rho})^{2}=\frac{R^{2}}{4 \pi b^{2}}
$$

as contribution of the TT polarization only. Here we have used the regular solution of eq. (4.19)

$$
\rho(\tau)=t_{b} \tau \Theta\left(b^{2}-\tau\right)+R^{2} \cosh ^{2} \chi(\tau) \Theta\left(\tau-b^{2}\right)
$$

where $\tau-b^{2}=R^{2}\left(\chi+\sinh \chi \cosh \chi-\chi_{b}-\sinh \chi_{b} \cosh \chi_{b}\right)$ and $t_{b} \equiv \tanh \chi_{b}$ is the largest real solution of eq. (5.1).

The complete result, including the $L T$ polarization would be [6]

$$
A_{R}=2 \pi G s\left(a_{0}+a_{H}+\cdots\right)=G s\left(\log \frac{L^{2}}{b^{2}}+\frac{R^{2}}{b^{2}}+\cdots\right)
$$

\footnotetext{
${ }^{10}$ This allows us to avoid an infrared divergence (present in eq. (4.27)) which is due to the $\varepsilon_{L T}$ polarization — and is to be subtracted out by the exponentiation procedure of ref [6].
} 


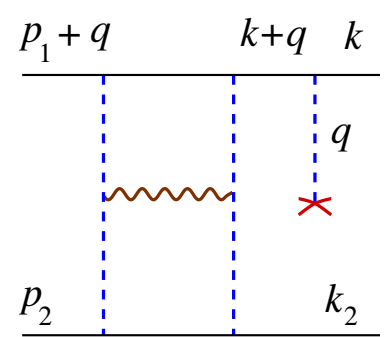

(a)

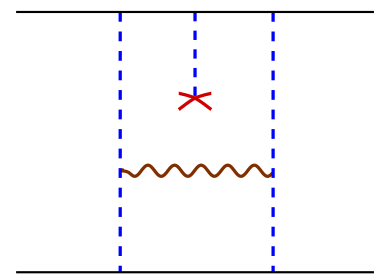

(b)

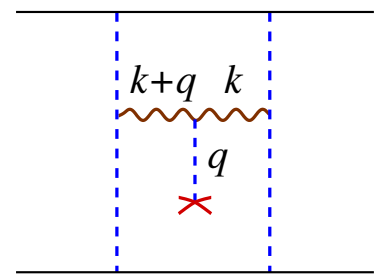

(c)

Figure 10. Insertions of the field $h_{--}$(red cross) on the H-diagram.

and provides — by stationarity of the eikonal phase — the scattering angle

$$
\sin \frac{\theta_{\mathrm{cl}}}{2}=-\frac{1}{\sqrt{s}} \frac{\partial}{\partial b} A_{R}=\frac{R}{b}\left[1+\left(\frac{R}{b}\right)^{2}+\cdots\right] .
$$

The recollection above suggests to generalize the calculation of the shifted field $h_{--}$in section 3 to the next order in the $R / b$ expansion, by including the H-diagram in the eikonal. In so doing we find 3 kinds of insertions of the $h_{--}$source, illustrated in figure 10: (a) insertions on the on-shell propagators, which can be done in a similar way as we did the leading one; (b) insertions on the particle propagators of the H-dagram itself, and (c) insertions on the emitted transverse field $h=\nabla^{2} \phi$. The latter are new and involve the rescattering vertex, which is omitted in the RAM calculation and will be included in the next subsection.

Insertions of type $(a)$ above add up to the leading shift $a_{0}(b)$ and are done by replacing $p^{+}$with $p^{+}+q^{+}$in the corresponding energy charge. ${ }^{11}$ However, Gs $a_{H} \sim G^{3} s^{2}$ contains two $p^{+}$factors and thus acquires a factor of 2 , as follows

$$
\begin{aligned}
A_{R} & \rightarrow 2 \pi G s\left[a_{0}\left(1+\frac{q^{+}}{\sqrt{s}}\right)+a_{H}\left(1+\frac{q^{+}}{\sqrt{s}}\right)^{2}\right] \\
& =2 \pi G s\left(a_{0}+a_{H}\right)+2 \pi R\left(a_{0}+2 a_{H}\right) \mathrm{i} \frac{\partial}{\partial x^{-}}+\cdots .
\end{aligned}
$$

The outcome is that the overall shift $2 \Delta$ is proportional to $\bar{a}(0)=a(b)$, as given by the perturbative formula (3.4):

$$
2 \Delta=2 \pi R\left(a_{0}+2 a_{H}+\cdots\right)=2 \pi R a(b)=\frac{\partial}{\partial \sqrt{s}} A_{R}(s, b)
$$

and thus it extends to the H-diagram the relationship between shift and energy-derivative of the action suggested in section 2 .

\footnotetext{
${ }^{11}$ We refer here to the overall shift $2 \Delta(\boldsymbol{x}, b)$ between past and future. Note that in the ACV equations framework the profile function $a(\boldsymbol{x})$ and the shift $\Delta(\boldsymbol{x}, b)$ occur together in the product $D(\boldsymbol{x}, b)$ (eq. (4.42)), while in the insertion formalism $\Delta(\boldsymbol{x}, b) \simeq \Delta(b)$ is probed by the leading profile $a_{0}(\boldsymbol{x})$ only, which dominates for $|\boldsymbol{x}| \gg b$.
} 


\subsection{Rescattering solutions with shifted fields}

Consider now the full field equations, starting from (4.12a). While, by replacing in the 1.h.s. the shock-wave (4.13), we generate terms proportional to $\kappa \sqrt{s} \delta\left(x^{-}\right)$, the rescattering term in the r.h.s. yields instead the structure

$$
-\kappa \sqrt{s}(2 \pi R)^{3} \delta^{\prime}\left(x^{-}\right) \frac{1}{2} \epsilon\left(x^{+}\right)\left|\partial^{* 2} \phi\right|^{2},
$$

which suggests an $x^{-}$translation, i.e., a shift similar to those just found at leading and H-diagram level with the eikonal insertions. Therefore, we make the Ansatz

$$
\begin{aligned}
\tilde{h}^{++} & =\kappa \sqrt{s} \delta\left(x^{-}-\pi R \Delta(\boldsymbol{x}) \epsilon\left(x^{+}\right)\right) a(\boldsymbol{x}) \\
\tilde{h}^{--} & =\kappa \sqrt{s} \delta\left(x^{+}-\pi R \bar{\Delta}(\boldsymbol{x}) \epsilon\left(x^{-}\right)\right) \bar{a}(\boldsymbol{x}) \\
\Phi & =\frac{\kappa^{3} s}{2} \phi(\boldsymbol{x})\left[\Theta\left(x^{-}-\pi R \Delta_{\phi}\right) \Theta\left(x^{+}-\pi R \bar{\Delta}_{\phi}\right)+\left(x^{ \pm} \rightarrow-x^{ \pm}\right)\right],
\end{aligned}
$$

where $\Delta, \bar{\Delta}, \Delta_{\phi}, \bar{\Delta}_{\phi}(\boldsymbol{x}, b)$ are shift variables to be determined. We take a similar Ansatz for energy-momentum too, because we know from last section the latter is really shifted at leading level. By then formally expanding the $\delta$-function (4.36a) in $R / x^{-}$, we get two equations from the zeros of the $\delta$ and $\delta^{\prime}$ contributions.

The first equation is simply the reduced-action model (RAM) equation for $a$, which reads

$$
|\partial|^{2} a+\frac{2 \pi R}{2}\left[\partial^{2}\left(\phi^{*} \partial^{* 2} a\right)-|\partial|^{2}\left(\phi^{*}|\partial|^{2} a\right)+\text { c.c. }\right]+\frac{1}{2} \delta^{2}(\boldsymbol{x})=0
$$

and reduces, in the axisymmetric case and for real-valued $\phi$, to

$$
\frac{\mathrm{d}}{\mathrm{d} r^{2}}\left[\dot{a} r^{2}\left(1-(2 \pi R)^{2} \dot{\phi}\right)\right]=\frac{\mathrm{d}}{\mathrm{d} r^{2}}[\dot{a} \rho]=-\frac{1}{2 \pi} \delta\left(r^{2}\right) .
$$

The latter is equivalent to eq. (4.19) and yields the solution

$$
a\left(r^{2}\right)=\frac{1}{2 \pi} \int_{r^{2}}^{L^{2}} \frac{\mathrm{d} r^{\prime 2}}{\rho\left(r^{\prime 2}\right)},
$$

where we have introduced the infrared cutoff parameter $L$ by setting $a\left(L^{2}\right)=0$. Since by eqs. (4.30) above [8]

$$
\rho\left(r^{2}\right) \simeq \begin{cases}r^{2}-R^{2} \log \frac{r^{2}}{4 b^{2}} & \left(r^{2} \gg b^{2}\right) \\ t_{b} r^{2} & \left(r^{2} \leq b^{2}\right)\end{cases}
$$

$a\left(r^{2}\right)$ approaches the leading value for $b \gg R$

$$
a\left(r^{2}\right) \simeq a_{0}\left(r^{2}\right)=\frac{1}{2 \pi} \log \frac{L^{2}}{r^{2}}+\cdots
$$

and diverges logarithmically for $r^{2} \rightarrow 0$.

The second equation (from the vanishing of the $\delta^{\prime}$-coefficient) involves the rescattering terms and is supposed to determine the shift parameter $\Delta$, which occurs in the combination $D(\boldsymbol{x}) \equiv \Delta(\boldsymbol{x}) a(\boldsymbol{x})$, as follows

$$
|\partial|^{2} D+\frac{(2 \pi R)^{2}}{2}\left[\partial^{2}\left(\phi^{*} \partial^{* 2} D\right)-|\partial|^{2}\left(\phi^{*}|\partial|^{2} D\right)+\text { c.c. }\right]=-\frac{\Delta_{0}}{2} \delta^{2}(\boldsymbol{x})+(2 \pi R)^{2}\left|\partial^{* 2} \phi\right|^{2},
$$


where we have set the rescattering terms on the r.h.s. and we notice in particular the energy-momentum shift which, according to section 3 , has the contribution

$$
\Delta_{0}=\bar{a}(0)=a(b) .
$$

emerging from the eikonal insertions up to $R^{2} / b^{2}$ accuracy. In the axisymmetric limit, eq. (4.42) takes the simplified form

$$
\begin{aligned}
\frac{\mathrm{d}}{\mathrm{d} r^{2}}(\rho \dot{D}) & =-\frac{\Delta_{0}}{2 \pi} \delta\left(r^{2}\right)+J_{\mathrm{R}}\left(r^{2}\right) \\
J_{\mathrm{R}}\left(r^{2}\right) & \equiv \frac{\Theta\left(r^{2}-b^{2}\right)}{(2 \pi R)^{2}}\left(\frac{\rho}{r^{2}}-\dot{\rho}\right)^{2}=(2 \pi R)^{2}\left(r^{2} \ddot{\phi}\right)^{2},
\end{aligned}
$$

where we note that the rescattering source $J_{\mathrm{R}}\left(r^{2}\right)$ vanishes for $r^{2} \leq b^{2}$, as a consequence of the equations of motion (4.40).

It is then straightforward to find a solution for $\Delta\left(r^{2}\right)$ which is regular everywhere, in the form

$$
\Delta\left(r^{2}\right)= \begin{cases}\Delta(\infty)+\frac{1}{a\left(r^{2}\right)} \int_{r^{2}}^{L^{2}} \frac{\mathrm{d} r^{\prime 2}}{\rho\left(r^{\prime 2}\right)} \int_{r^{\prime 2}}^{\infty} \mathrm{d} r^{\prime \prime 2} J_{\mathrm{R}}\left(r^{\prime \prime 2}\right) & \left(r^{2} \geq b^{2}\right) \\ \Delta_{0}-\frac{1}{a\left(r^{2}\right)}\left[2 \pi a(b) \int_{b^{2}}^{\infty} \mathrm{d} r^{\prime 2} J_{\mathrm{R}}\left(r^{\prime 2}\right)-\int_{b^{2}}^{L^{2}} \frac{\mathrm{d} r^{\prime 2}}{\rho\left(r^{\prime 2}\right)} \int_{r^{\prime 2}}^{\infty} \mathrm{d} r^{\prime \prime 2} J_{\mathrm{R}}\left(r^{\prime \prime 2}\right)\right] & \left(r^{2} \leq b^{2}\right)\end{cases}
$$

where

$$
\Delta(\infty)=\Delta_{0}-2 \pi \int_{b^{2}}^{\infty} \mathrm{d} r^{2} J_{\mathrm{R}}\left(r^{2}\right)=a(b)-\int_{0}^{\infty} \frac{\mathrm{d} r^{2}}{2 \pi R^{2}}(1-\dot{\rho})^{2} .
$$

In other words, the shift parameter of the longitudinal field takes over the (constant) leading value $a(b)$ from the energy-momentum tensor and adds an $r^{2}$-dependent term of relative order $R^{2} / b^{2}$ which, for $r^{2} \gg b^{2}$, is related to the kinetic term of the RAM action

$$
A_{R}=2 \pi G s\left[a(b)-\int_{0}^{\infty} \frac{\mathrm{d} r^{2}}{2 \pi R^{2}}(1-\dot{\rho})^{2}\right] \equiv 2 \pi G s \mathcal{A}(b) .
$$

This means that, while the RAM action is sufficient in order to describe the scattering parameters at relative order $R^{2} / b^{2}$ (H diagram), the rescattering terms are needed in order to describe the form of the fields, like shock-wave shifts, and thus the metric properties at a comparable level of accuracy.

It is amusing to check the result (4.46) - which is based on the rescattering equations and on the energy momentum shift in eq. (4.42) - by using the direct insertions (b) and (c) on the H-diagram mentioned before, and depicted in figure 10. By keeping track of the flow of energy charges we calculate the above insertions on the imaginary parts (appendix A) and we find that diagram (b) is already counted by Lipatov's vertices, while diagram $(c)$ contributes the absorptive part

$$
\kappa \sqrt{s} G s \frac{R^{2}}{2} \int \frac{\mathrm{d}^{2} \boldsymbol{q}}{2 \pi^{2} \boldsymbol{q}^{2}} \mathrm{e}^{\mathrm{i} \boldsymbol{q} \cdot \boldsymbol{x}} \int \mathrm{d}^{2} \boldsymbol{k} \tilde{h}(\boldsymbol{k}) \tilde{h}^{*}(\boldsymbol{k}+\boldsymbol{q}) \int \frac{\mathrm{d} q^{+}}{4 \pi} \int \mathrm{d} k^{+} \frac{q^{+}}{k^{+}} \mathrm{e}^{-\mathrm{i} \frac{q^{+}}{2} x^{-}} \Theta\left(x^{+}\right) .
$$


The $q^{+}$-dependent part, by dividing out the rapidity factor $(2 / \pi) \int \mathrm{d} k^{+} / k^{+}=2 Y / \pi$ (as requested in order to obtain the real part of the diagram from the dispersion relations) and the leading field $a_{0}\left(r^{2}\right)$ fits with the displacement form i $\left(\Delta_{R}+\mathrm{i} \Delta_{I}\right) \mathrm{i} \partial_{-}=-\left(\Delta_{R}+\mathrm{i} \Delta_{I}\right) \partial_{-}$, by thus providing the shift $2 \pi R \Delta_{H}$ from past to future, where

$$
\Delta_{R}=\Delta_{H}=-\frac{1}{a_{0}\left(r^{2}\right)} \frac{(\pi R)^{2}}{2} \int \mathrm{d}^{2} \boldsymbol{x}^{\prime}\left|h_{0}\left(\boldsymbol{x}^{\prime}\right)\right|^{2} \frac{\mathrm{d}^{2} \boldsymbol{q}}{2 \pi^{2} \boldsymbol{q}^{2}} \mathrm{e}^{\mathrm{i} \boldsymbol{q} \cdot\left(\boldsymbol{x}-\boldsymbol{x}^{\prime}\right)} .
$$

We recognize here the 2-dimensional Laplacian Green function $G_{0}\left(\boldsymbol{x}-\boldsymbol{x}^{\prime}\right)$ applied to the H-diagram density. By translating it to the azimuthal-averaged formalism we can use the one-dimensional form

$$
G_{0}\left(r, r^{\prime}\right)=\frac{1}{2 \pi} \log \frac{L^{2}}{r_{>}^{2}}, \quad\left(r_{>} \equiv \max \left(r, r^{\prime}\right)\right)
$$

and apply it to the rescattering current $J_{\mathrm{R}}\left(r^{2}\right)$. The result is just identical to the r.h.s. of eq. (4.46) with the $\Delta_{0}$ contribution subtracted out. In particular, for $r \gg b$ eq. (4.50) factorizes and yields $\Delta_{H}(\infty)=-a_{H}$, thus recovering the form (4.48) of the total shift.

Our overall interpretation of the present findings is that a large-distance observer will see the particle shock-waves to suffer a total time delay $2 \pi R \mathcal{A}(b)$, directly related to the action and thus to the scattering angle

$$
\sin \frac{\theta_{\mathrm{cl}}(b)}{2}=-\pi R \frac{\partial}{\partial b} \mathcal{A}(b)=-\frac{1}{\sqrt{s}} \frac{\partial}{\partial b} A_{R}=\frac{b R}{\rho\left(b^{2}\right)}
$$

because of the contribution of the rescattering shift. On the other hand, a test particle parallel to particle 2 , traveling at transverse distance $r \simeq b$ from particle $1^{12}$ will see a profile function $2 \pi R a\left(r^{2}\right)$ related to its own scattering angle

$$
\sin \frac{\theta(r)}{2}=-\pi R \frac{\partial}{\partial r} a\left(r^{2}\right)=\frac{r R}{\rho\left(r^{2}\right)} \stackrel{r \rightarrow b}{\longrightarrow} \sin \frac{\theta_{\mathrm{cl}}}{2}
$$

which turns out to be the same in the limit $r=b .{ }^{13}$ Furthermore, the test-particle will suffer a time delay that, due to the double-shift picture of section 2.3, is $\Delta t-\Delta z=2 \pi R a(x)$, close to the short-distance value $2 \pi R a(b)=\partial A_{R} / \partial \sqrt{s}$ coming from the eikonal insertions of section 3

Finally, while the rescattering contribution is needed for the large distance shifts, its contribution to the action starts at order $(R / b)^{4}$ because the diagram in figure 9.c (formally of order $\left.(R / b)^{3}\right)$ vanishes by the property $\epsilon\left(x^{+}\right) \delta\left(x^{+}\right)=0$. Therefore it does not affect the consistency of the metric and action descriptions at order $(R / b)^{2}{ }^{14}$

Of course, a symmetrical calculation yields the shift $\pi R \epsilon\left(x^{-}\right) \bar{\Delta}(\boldsymbol{x})$ for the field $h_{++}$, provided one exchanges light-cone indices $+\leftrightarrow-$ and the profile functions and shifts $a \leftrightarrow \bar{a}, \Delta \leftrightarrow \bar{\Delta}$.

\footnotetext{
${ }^{12}$ We mean $r=b$ at deflection time, corresponding to the perpendicular distance (impact parameter) $b_{0}=b \cos (\theta / 2)$, according to the discussion of section 2.2 .

${ }^{13}$ That is because the $b$-derivative of $A_{R}(b)$ has implicit contributions which vanish by the equation of motion.

${ }^{14}$ The absence of corrections of relative order $(R / b)^{3}$ was argued $[1-3,7]$ to vanish for light-particle scattering by analiticity and relativity arguments.
} 


\subsection{Shift modification for the transverse field}

We now go over to the second basic equation (4.12b) which essentially describes rescattering properties (figure 9.c,d) of the transverse field $h=\nabla^{2} \phi$, once emitted by the longitudinal fields. We note that, by replacing the RAM fields (4.36) in the r.h.s. of eq. (4.12b), we obtain the structure

$$
\begin{aligned}
\kappa(\kappa \sqrt{s})^{2} & {\left[\partial^{* 2}\left(\bar{a} \partial^{2} \phi\right)+\text { c.c. }\right] \times } \\
& \times\left\{-\frac{\partial}{\partial \Delta} \frac{1}{2}\left[\delta\left(x^{-}-\pi R \Delta\right) \delta\left(x^{+}-\pi R \bar{\Delta}_{\phi}\right)+\delta\left(x^{-}+\pi R \Delta\right) \delta\left(x^{+}+\pi R \bar{\Delta}_{\phi}\right)\right]\right\} .
\end{aligned}
$$

Let us recall that, according to eqs. (4.43), (4.46), the longitudinal rescattering predicts a shift $\Delta=a(b)$. We then decide to expand the shift $\Delta_{\phi}$ of the transverse field around the constant value $\Delta=a(b) \neq 0$, which gives the leading order of the longitudinal shift. By this method, it is easy to evaluate a (formally) first order modification $\Delta^{(1)} \equiv \Delta-\Delta_{\phi}$ of the transverse-field shift, which however is also of leading order in the $R / b$ expansion. By expanding the first term in the l.h.s. of eq. (4.12b) we obtain

$$
\begin{aligned}
\partial_{-} \partial_{+}|\partial|^{4} \Phi= & |\partial|^{4} \phi\left[1-\Delta^{(1)} \frac{\partial}{\partial \Delta}-\bar{\Delta}^{(1)} \frac{\partial}{\partial \bar{\Delta}}+\cdots\right] \times \\
& \times \frac{1}{2}\left[\delta\left(x^{-}-\pi R \Delta\right) \delta\left(x^{+}-\pi R \bar{\Delta}\right)+\delta\left(x^{-}+\pi R \Delta\right) \delta\left(x^{+}+\pi R \bar{\Delta}\right)\right]
\end{aligned}
$$

so that we can easily match the left and the r.h.s. to get the equation, in the axisymmetric limit,

$$
2|\partial|^{4}\left(\phi \Delta^{(1)}\right)=\partial^{* 2}\left(\bar{a} \partial^{2} \phi\right)+\text { c.c. },
$$

where we work in the regime $r \gg b$ with $\bar{a}\left(r^{2}\right)=a\left(r^{2}\right) \simeq \frac{1}{2 \pi} \log \frac{L^{2}}{r^{2}}$.

The expression of $\phi$ is found by the RAM equation

$$
-2 \frac{\mathrm{d}}{\mathrm{d} r^{2}} \frac{r^{2} \ddot{\rho}}{(2 \pi R)^{2}}=2|\partial|^{4} \phi=\partial^{* 2} a \partial^{2} \bar{a}-|\partial|^{2} a|\partial|^{2} \bar{a}=-\frac{\mathrm{d}}{\mathrm{d} r^{2}}\left(r^{2} \dot{a} \dot{\bar{a}}\right),
$$

which was provided before (4.19). By inserting proper boundary conditions, in our regime $r \gg b$ we can set $\phi=\frac{1}{4}\left[a\left(r^{2}\right)-a\left(b^{2}\right)\right]^{2}$ and tentatively look for a solution $\Delta^{(1)}=\lambda a\left(r^{2}\right)+\mu$, where $\lambda$ and $\mu$ are constants. A simple calculation shows that

$$
\Delta^{(1)}=\frac{2}{3} a\left(r^{2}\right)+\frac{1}{3} a\left(b^{2}\right)-\frac{1}{2 \pi}, \quad \Delta_{\phi}=\frac{2}{3}\left[a\left(b^{2}\right)-a\left(r^{2}\right)\right]+\frac{1}{2 \pi},
$$

so that $\Delta_{\phi}$ is $r^{2}$-dependent and of leading order, while the correction $\Delta^{(1)}$ is sizeable. Furthermore, $\Delta_{\phi}$ becomes cutoff independent, and pretty small at $r=b$.

The above conclusion may be unpalatable from a calculational standpoint, but is natural on physical grounds because rescattering occurs for the $\phi$ field at relatively leading level and at all distances, so that the parameter $a(b)$ has no particular role and a sizeable difference $\Delta-\Delta_{\phi}$ is expected. One might ask, at this point, whether the RAM hierarchy for the action is really satisfied or not. Fortunately it remains, because the integral over $x^{+}, x^{-}$of the rescattering vertex with the longitudinal field just vanishes $\left(\epsilon\left(x^{-}\right) \delta\left(x^{-}\right)=0\right)$ 
or, in other words, there is no $R^{3} / b^{3}$ contribution to the action from diagram 9.c. Furthermore, the shift does not change the $x^{+}, x^{-}$integration in the H-diagram and therefore rescattering contributions.

On the other hand, the location of the shock-wave and the evolution of particle trajectories and geodesics does change from the point of view of the metric, so that rescattering is needed in order to have self-consistent calculations even at relative order $R^{2} / b^{2}$.

\section{$5 \quad$ Irregular solutions and their (re)scattering properties}

All preceding arguments hold for the UV-safe solutions of the RAM model in eqs. (4.18), (4.19) which, by definition satisfy the condition $\rho(0)=0$. Due to the form of $\rho\left(r^{2}\right)$ in eq. (4.17), $\rho(0) \neq 0$ would imply that $\dot{\phi} \sim-\rho(0) / r^{2}$ has a short-distance singularity, $\boldsymbol{\nabla} \phi$ has an outgoing flux $-\rho(0)$ and therefore $h=\nabla^{2} \phi \sim-\rho(0) \delta\left(r^{2}\right) /(\pi R)^{2}$ has a singular $\delta$-function contribution. The latter behaviour would call for large short-distance effects - possibly regularized by the string — which are expected, but not considered, in the RAM model.

Restricting the solutions by $\rho(0)=0$ is possible only if the impact parameter is larger than some critical value $b_{c} \sim R$ which signals a possible classical collapse. In fact, the regular solution (4.30) of eq. (4.19) has continuous derivative at $\tau \equiv r^{2}=b^{2}$ provided

$$
t_{b}\left(1-t_{b}^{2}\right)=\frac{R^{2}}{b^{2}}
$$

a condition which has real-valued solutions only for $b^{2} \geq b_{c}^{2}=(3 \sqrt{3} / 2) R^{2}$. On the other hand, the complex solutions for $b<b_{c}$ lead to an exponential damping [9] of the $S$-matrix of type $\sim \exp (-G s)$ - with exponent of the order of a black-hole entropy $E R$ - which leads, eventually, to a violation of unitarity of the RAM model [10, 14].

From the above discussion, some questions arise: what happens to the rescattering solutions for $b<b_{c}$, when they are irregular? Do we see any sign of a possible collapse in the latter, perhaps in relation with the unitarity problem and to a "fall in the center" mechanism [20]?

Let us then consider a class of real-valued RAM solutions with $\rho(0) \neq 0$ for $b<b_{c}$, as follows. We start from the general solution for $\rho(0) \neq 0$

$$
\rho(\tau)=\left[\rho(0)+t_{b} \tau\right] \Theta\left(b^{2}-\tau\right)+R^{2} \cosh ^{2} \chi(\tau) \Theta\left(\tau-b^{2}\right)
$$

where $t_{b}$ is now a free parameter which $\rho(0)>0$ depends on. Then we choose $t_{b} \equiv t_{m}$ so as to minimize $\rho(0)$, i.e., $\mathrm{d} \rho(0) /\left.\mathrm{d} t_{b}\right|_{t_{m}}=0$. The parameters of this "minimal" solution $\rho_{m}(\tau)$ are given by

$$
\frac{\left(1-t_{m}^{2}\right)^{2}}{2 t_{m}}=\frac{R^{2}}{b^{2}}, \quad \rho_{m}(0)=\frac{R^{2}}{1-t_{m}^{2}}-t_{m} b^{2}=R^{2} \frac{1-3 t_{m}^{2}}{\left(1-t_{m}^{2}\right)^{2}} .
$$

The corresponding rescattering current is

$$
J_{\mathrm{R}}(\tau)=(2 \pi R)^{2}(\tau \ddot{\phi})^{2}=\frac{1}{(2 \pi R)^{2}}\left(\dot{\rho}-\frac{\rho}{\tau}\right)^{2} \stackrel{\tau<b^{2}}{\longrightarrow} \frac{1}{(2 \pi R)^{2}} \frac{\rho^{2}(0)}{\tau^{2}} .
$$


The above current is now non-vanishing for $\tau<b^{2}$, and, in addition, has a non-integrable behaviour for $\tau \rightarrow 0^{+}$. This means that large rescattering amplitudes are built in the short-distance region $\lambda_{s}<r, b<R$ and, as a consequence, that exchange and emission of (massive) string states can no longer be neglected.

Nevertheless, we find it instructive to provide here a preliminary analysis of irregular solutions in our effective-theory framework in which only graviton intermediate states are considered. Basic string effects - like graviton reggeization and ensuing string production at $\lambda_{s}[1-3]$, as well as diffractive and central string emission induced by tidal forces [1$3,21]^{15}$ should certainly be estimated in the near future in order to see how they affect the picture, but we feel that our effective approach may still provide suggestions and questions to be answered, and is anyway needed as a ground for the estimates just mentioned.

In order to get a better insight on the evolution of irregular solutions, we cut-off the current (5.4) below $r=\lambda_{s} \ll R$, and we compute the total charge, related in section 4 to the H-diagram action $2 \pi G s a_{H}$ and to the shift $\Delta_{H}$ as follows:

$$
\begin{aligned}
a_{H} & =-\Delta_{H}=2 \pi \int_{\lambda_{s}^{2}}^{\infty} J_{\mathrm{R}}(\tau) \mathrm{d} \tau \\
& =\frac{1}{2 \pi R^{2}}\left[\int_{0^{+}}^{\infty}(1-\dot{\rho})^{2} \mathrm{~d} \tau-2\left[1-\dot{\rho}\left(0^{+}\right)\right] \rho(0)+\frac{\rho^{2}(0)}{\lambda_{s}^{2}}+\mathcal{O}\left(\lambda_{s}^{2}\right)\right],
\end{aligned}
$$

where the singular integration has been performed by a careful integration by parts and in the last equality we have introduced the $\delta$-function singularity in the $\rho$-derivative:

$$
\dot{\rho}(\tau)=\left.\dot{\rho}(\tau)\right|_{\mathrm{reg}}+\rho_{m}(0) \delta(\tau), \quad \nabla^{2} \phi=\frac{1-\left.\dot{\rho}(\tau)\right|_{\mathrm{reg}}}{(\pi R)^{2}}-\frac{\rho(0) \delta(\tau)}{(\pi R)^{2}} .
$$

This means that we think of $\rho(\tau)=0$ for $\tau \leq 0$ (a region which is felt by the twodimensional model through the outgoing flux) and that the $\delta$-function occurs because of the $\tau$-discontinuity.

Thus, strictly speaking, we discover that the singular solutions are not bona fide solutions of the equations of motion up to $\tau=0^{-}$unless we introduce a singular "external force" $\ddot{\rho}=\rho(0) \delta^{\prime}(\tau)$ at $\tau=0$. We can do that by starting from the action [14]

$$
\frac{A}{2 \pi G s}=\int \mathrm{d}^{2} \boldsymbol{x}\left[a \bar{s}+\bar{a} s-\frac{1}{2} \boldsymbol{\nabla} a \cdot \boldsymbol{\nabla} \bar{a}-(\pi R)^{2}\left(\frac{1}{2}\left(\boldsymbol{\nabla}^{2} \phi\right)^{2}+\boldsymbol{\nabla} \phi \cdot \nabla \mathcal{H}\right)\right]
$$

and by supplementing the Lipatov's current $\mathcal{H}$ of eq. (4.15) with a singular external current

$$
\delta \dot{\mathcal{H}}=\dot{\mathcal{H}}+2 \dot{a} \dot{\bar{a}}=-\frac{\rho_{m}(0)}{(\pi R)^{2}} \delta^{\prime}(\tau) .
$$

By then replacing such expression into eq. (5.7), i.e.,

$$
-(\pi R)^{2} \boldsymbol{\nabla} \phi \cdot \nabla \mathcal{H}=-[\rho(\tau)-\tau]\left(2 \dot{a} \dot{\bar{a}}+\frac{\rho_{m}(0) \delta^{\prime}(\tau)}{(\pi R)^{2}}\right),
$$

\footnotetext{
${ }^{15}$ Tidal-force production amplitudes are determined by the second $(b, r)$-derivatives of phaseshifts [1$3,21]$ and are thus possibly important in the whole subcritical region $\lambda_{s}<r, b<R$ where (5.4) is large, so that unitarity defect $[10,14]$ and energy balance $[22,23]$ could be sizeably affected.
} 
we find that the action enforces eq. (5.6) and becomes, on the equation of motion,

$$
\begin{aligned}
\frac{A}{2 \pi G s} & =\pi\left\{\int[a \bar{s}+\bar{a} s-2 \rho \dot{a} \dot{\bar{a}}] \mathrm{d} \tau-\frac{1}{2} \int_{0^{+}}^{\infty}\left(\frac{1-\dot{\rho}}{\pi R}\right)^{2} \mathrm{~d} \tau+\frac{1}{2}\left(\frac{\rho_{m}(0)}{\pi R}\right)^{2} \delta(0)\right\} \\
& =a(b)-\int_{0^{+}}^{\infty} \frac{(1-\dot{\rho})^{2}}{2 \pi R^{2}} \mathrm{~d} \tau+\frac{\rho_{m}^{2}(0)}{2 \pi R^{2} \lambda_{s}^{2}}
\end{aligned}
$$

thus determining the singular term in the action. ${ }^{16}$

We are now able to look at the scattering and shift properties of the irregular solutions, by keeping in mind that we need in this case the external current (5.8), that we think generated by the short-distance string dynamics and/or by possibly collapsed matter. Compared to the complex solutions - which have a quantum-tunneling interpretation and cause a probability suppression - the irregular ones may provide a probability source or alternatively may carry away the information loss.

By thus using the eikonal representation of the $S$-matrix with the action (5.10) and by carefully computing $b$ - and $\sqrt{s}$-derivatives, we get the scattering angle

$$
\pm \sin \frac{\theta_{s}}{2}=-\frac{1}{\sqrt{s}} \frac{\partial A}{\partial b}=\frac{R b}{\rho\left(b^{2}\right)}+\pi^{2} R \mathcal{H}(0) \frac{\partial \rho(0)}{\partial b}
$$

and the time delay

$$
t_{D}=\frac{\partial A}{\partial \sqrt{s}}=2 \pi R\left[a(b)-\frac{\pi}{2} \mathcal{H}(0) \sqrt{s} \frac{\partial \rho(0)}{\partial \sqrt{s}}\right] \equiv 2 \pi R \Delta_{0},
$$

where, by eqs. (5.2), (5.8)

$$
\mathcal{H}(0)=\frac{1}{(\pi R)^{2}}\left[1-\dot{\rho}\left(0^{+}\right)-\frac{\rho(0)}{\lambda_{s}^{2}}\right] .
$$

Such results can be further specified by using

$$
\frac{\partial \rho(0)}{\partial b}=-2 t_{m} b=-\left(\frac{R b}{\rho\left(b^{2}\right)}\right)^{2} b, \quad \frac{\sqrt{s}}{2} \frac{\partial \rho(0)}{\partial \sqrt{s}}=\frac{R^{2}}{1-t_{m}^{2}}=\rho\left(b^{2}\right)
$$

and the outcomes are plotted in figure 11.

We note the strong increase of the action in the region $b \simeq \lambda_{s}$ due to the positive singular contribution - implying in particular a motion with many turns in the region $\lambda_{s} \lesssim b \lesssim R$. Actually, large scattering angles are reached pretty soon around $b=b_{c}$ as solutions of the equation

$$
\pm \sin \frac{\theta_{s}}{2}=\left(\frac{b}{R}\right)^{3} \frac{R^{2}}{\lambda_{s}^{2}}\left[1-3 t_{m}^{2}\left(\frac{b}{R}\right)\right],
$$

but, due to the strong increase of the action derivative (figure 11), the saddle-points are confined to either the critical region $b_{c}^{2}-b^{2}=\mathcal{O}\left(\lambda_{s}^{2}\right)$ or to the small- $b$ region $(b / R)^{3}=$

\footnotetext{
${ }^{16}$ Note that we interpret the distribution $\delta(\tau)$ as a step-function located around $\tau=0$ with width $\lambda_{s}^{2}$ and height $\lambda_{s}^{-2}$, hence the identification $\delta(0)=\lambda_{s}^{-2}$.
} 

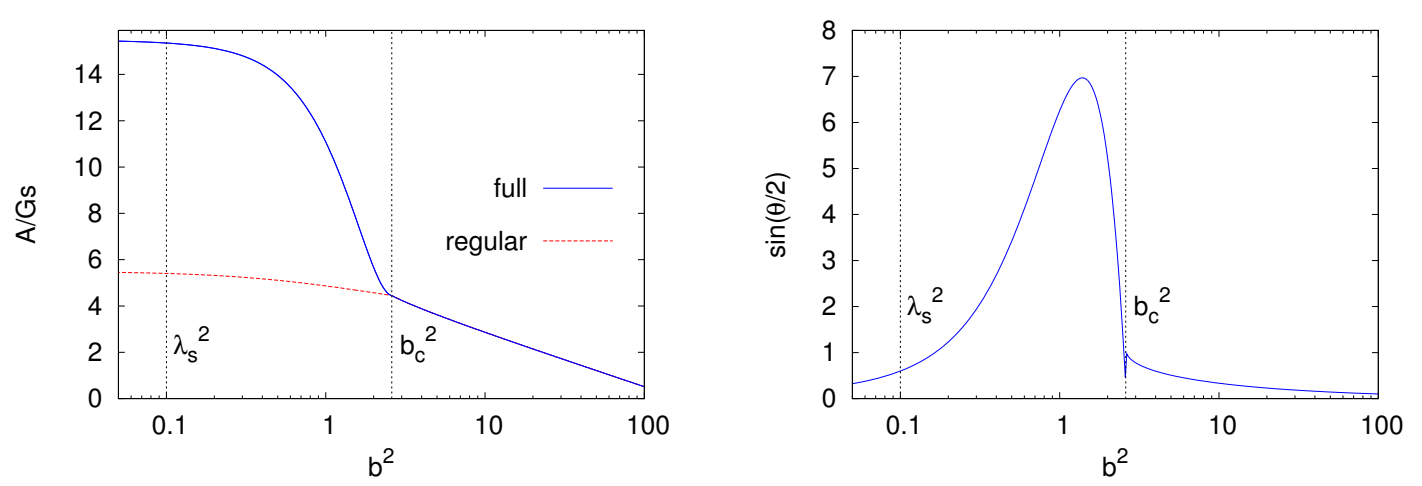

Figure 11. (a) action of the solutions of equation of motion (blue solid line); the red dashed line shows the action for $b<b_{c}$ without the $\rho(0)$ contribution; (b) scattering angle as derived by eq. (5.11). In both plots $\lambda_{s}^{2}=0.1 R^{2}$; lengths are measured in units of $R=1$.

$\mathcal{O}\left(\lambda_{s}^{2} / R^{2}\right)$, for all real values of $\theta_{s}{ }^{17}$ Finite $b$-values, in the region $\lambda_{s}^{2} \ll b^{2}<b_{c}^{2}-\lambda_{s}^{2}$ are strongly suppressed like $\exp \left(-G s R^{2} / \lambda_{s}^{2}\right)$.

The above observation suggests that the singular solutions under study do not actually yield back all the initial information in the physical scattering region, but may carry it away in the small- $b$, string-dominated region.

Another piece of information comes from the study of the shifts and the corresponding time delay. We first notice, by eq. (5.12), that the short-distance shift of

$$
\Delta_{0}=a(b)+\left[\frac{\rho_{m}(0)}{\lambda_{s}^{2}}-\left(1-t_{m}\right)\right] \frac{2 \rho\left(b^{2}\right)}{2 \pi R^{2}}
$$

has a positive singular term - meaning a lot of time spent in the interaction region which is essentially twice the singular contribution to $a_{H}$ in eq. (5.5). The factor of 2 comes from $\rho(0)$ scaling like $R^{2} \sim(G \sqrt{s})^{2}$ - similar to what happens for the H-diagram itself.

Furthermore, we already know from section 4 that the large-distance shift takes contributions from rescattering insertions and produces the additional (negative) shift $\Delta_{H}$, the one we started with in eq. (4.34). We thus have

$$
\begin{aligned}
\Delta(\infty) & =\Delta_{0}+\Delta_{H}=a(b)-\int_{0^{+}}^{\infty} \frac{(1-\dot{\rho})^{2}}{2 \pi R^{2}} \mathrm{~d} \tau+\frac{1}{2 \pi R^{2}} \frac{\rho_{m}^{2}(0)}{\lambda_{s}^{2}}-\mathcal{H}(0) \pi t_{m} b^{2} \\
& =\mathcal{A}\left(b ; \lambda_{s}\right)-\mathcal{H}(0) \pi t_{m} b^{2}
\end{aligned}
$$

We note that the singular term remains positive after subtraction of $\Delta_{H}$ and of the same order as that of the action - apart for the addition of a term proportional to $-\mathcal{H}(0)>0$. The very large time delay is thus confirmed at all distances. That means that the singular solutions, wherever they are, spend a long time in the interaction region, before exiting, either around $b=b_{c}$, or at $b=\mathcal{O}\left(\lambda_{s}\right)$. Therefore, they are connected with long-lived states in that region.

\footnotetext{
${ }^{17}$ We have checked that complex saddle-points do not change the picture.
} 
Finally, let us look at the associated metric (4.21), whose geodesics - in the "regular" case $\rho(0)=0$ - were argued to provide a complementary picture of particle scattering. For the irregular solutions of eqs. (5.2), (5.3), the relevant metric coefficients (inside the light-wedges $x^{+} x^{-}>0$ ) have the form

$$
\begin{aligned}
& G_{+-} \equiv-2 g_{+-}=1-\frac{1}{2}(1-\dot{\rho}) \quad \longrightarrow 1 \\
& G_{r r} \equiv g_{r r}=1+\frac{1}{2}(1-\dot{\rho})-\frac{1}{2}\left(\frac{\rho}{r^{2}}-\dot{\rho}\right) \quad \longrightarrow 1-\frac{r^{* 2}}{r^{2}} \quad\left(r^{* 2} \simeq \frac{\rho(0)}{2}\right) \\
& G_{\theta \theta} \equiv \frac{g_{\theta \theta}}{r^{2}}=1+\frac{1}{2}(1-\dot{\rho})+\frac{1}{2}\left(\frac{\rho}{r^{2}}-\dot{\rho}\right) \longrightarrow 1+\frac{r^{* 2}}{r^{2}},
\end{aligned}
$$

where the arrows label the simplified expressions obtained in the $\dot{\rho} \rightarrow 1$ limit, which will be used for the purpose of the qualitative discussion below.

We note that, for $\rho(0) \neq 0, g_{r r}$ shows a puzzling zero at $r^{2}=r^{* 2} \sim \rho(0) \sim R^{2}$, which causes a change of signature of the $r$-dimension for $r<r^{*}$ - a feature to be taken with great caution because our perturbative identification of the metric coefficient is probably invalid in the strong-coupling region we are interested in. Nevertheless, let us take the expressions (5.18) at face-value, and discuss the ensuing geodetic flow, in the transverse plane, which is strongly affected by the $\phi$-field singularity $\dot{\phi} \sim-\rho(0) / r^{2}$.

In order to do that, we shall introduce the test-particle lagrangian (per unit mass)

$$
L=-\frac{|\mathrm{d} s|}{\mathrm{d} t}=-\sqrt{G_{+-}\left(1-\dot{z}^{2}\right)-G_{r r} \dot{r}^{2}-G_{\theta \theta} r^{2} \dot{\theta}^{2}}
$$

with corresponding momenta and hamiltonian (per unit mass)

$$
\begin{aligned}
P_{z} & \equiv \frac{\mathrm{d} L}{\mathrm{~d} \dot{z}}=\frac{G_{+-}}{-L} \dot{z}=\text { const } \\
P_{\theta} & \equiv \frac{\mathrm{d} L}{\mathrm{~d} \dot{\theta}}=\frac{G_{\theta \theta}}{-L} r^{2} \dot{\theta}=\mathrm{const} \\
P_{r} & \equiv \frac{\mathrm{d} L}{\mathrm{~d} \dot{r}}=\frac{G_{r r}}{-L} \dot{r} \\
H & \equiv P_{z} \dot{z}+P_{\theta} \dot{\theta}+P_{r} \dot{r}-L=\frac{G_{+-}}{-L}=\text { const } .
\end{aligned}
$$

It is convenient to define the momenta per unit energy $p_{i} \equiv P_{i} / H$, so as to allow the treatment of the massless case too. By evaluating the constants of motion in the asymptotic region $r \rightarrow \infty$ one finds that $p_{z}=\dot{z}=v_{z}, H=\left(1-v^{2}\right)^{-1 / 2}$ and $p_{\theta}=\beta v_{\perp}$, where $v_{z}, v_{\perp}$ and $v=\sqrt{v_{z}^{2}+v_{\perp}^{2}}$ are the longitudinal, transverse and total (asymptotic) velocity, while $\beta$ is the impact parameter of the test particle w.r.t. the $z$ axis.

The test-particle motion is eventually described by the constants of motion $p_{z}, p_{\theta}, H$ and by the radial "effective potential"

$$
-p_{r}^{2}=-\frac{G_{r r}}{G_{+-}}\left[1-v_{z}^{2}-\left(1-v^{2}+\frac{\left(\beta v_{\perp}\right)^{2}}{G_{\theta \theta} r^{2}}\right) G_{+-}\right] \rightarrow-\left(1-\frac{r^{* 2}}{r^{2}}\right) v_{\perp}^{2}\left(1-\frac{\beta^{2}}{r^{2}+r^{* 2}}\right),
$$

since $\dot{r}^{2}-p_{r}^{2}\left(G_{+-} / G_{r r}\right)^{2}=0$. 


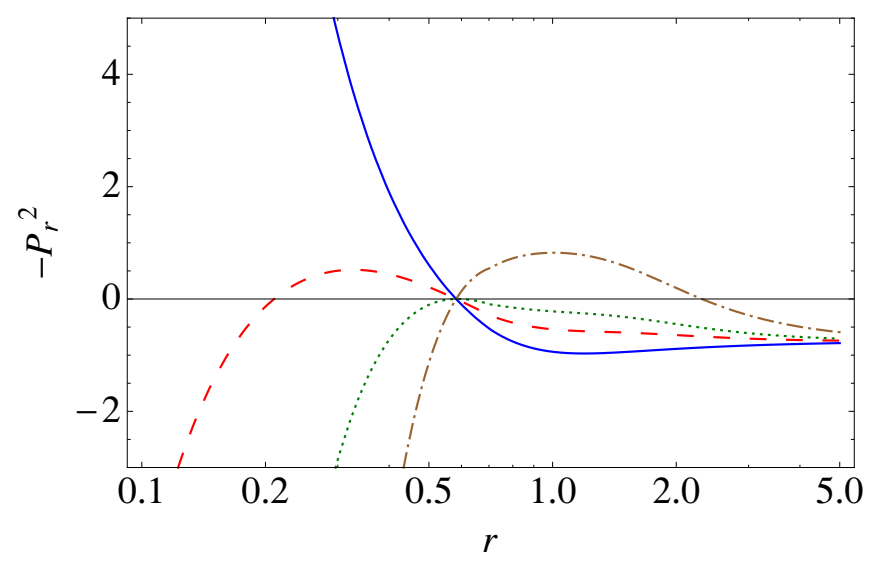

Figure 12. Radial "effective potential" for different values of the test-particle's impact parameter: $|\beta|<r^{*}$ (solid blue), $r^{*}<|\beta|<\sqrt{2} r^{*}$ (dashed red), $|\beta|=\sqrt{2} r^{*}$ (dotted green), $|\beta|>\sqrt{2} r^{*}$ (dash-dotted brown).

The $\beta=0$ geodesics are characterized by the fact that the transverse velocity $v_{\perp}$ (if present) is purely radial and the potential (5.21) is purely repulsive (figure 12), and would be typical of an angular momentum barrier with $\left|p_{\theta} / v_{\perp}\right|=|\beta|=r^{*}$ that - by lack of other explanations - we should attribute to the external source $\delta \mathcal{H}$ in eq. (5.8) that we have put in at $r=0$ in order to justify the irregular solutions themselves. For $v_{\perp}=0$ the repulsive potential is absent, and the geodesics (at $x^{-}=0$, say) will reach the shock-wave (at $x^{+}=0$, say) at the initial distance $r$, but will thereby acquire a shift and will be deflected according to the formula

$$
\tan \frac{\theta_{f}}{2}=\frac{1}{\sqrt{1-\frac{r^{*} 2}{r^{2}}}}\left(\tan \frac{\theta_{i}}{2}-\pi R \frac{\mathrm{d} \bar{a}(r)}{\mathrm{d} r}\right),
$$

which takes into account the "refraction index" of the $\phi$-field. Once again $r=r^{*}$ is unreachable: if initially we try to approach it, the geodesic scattering angle approaches $\pi$, and the test particle is reflected backwards.

We note at this point that the situation does change - unexpectedly - when $|\beta|$ increases and overcomes the threshold $r^{*}$ : in this case, according to eq. (5.21), the centrifugal factor at the origin changes sign, so that the potential becomes partly attractive, close to the origin and above $r^{*}$ (figure 12). In particular, for $|\beta|=\sqrt{2} r^{*}$ the potential is everywhere attractive!

This somewhat surprising feature opens up the possibility that test particles may reach the small- $r$ region, at least at quantum level, because the small barrier has a transmission coefficient of order unity around the value $|\beta|=\sqrt{2} r^{*}$, and is thus able to populate the small- $r$ region around $\lambda_{s}$ in an efficient way. That feature is the counterpart of the selection of possible issues of the particles themselves, which was argued before to have sizeable probability for a small- $b$ exit on the basis of the action stationarity condition. Furthermore, the test-particle action variable $\frac{1}{p_{\perp}} \int_{\lambda_{s}}^{r^{*}} p_{r} \mathrm{~d} r \sim \sqrt{2} r^{*} \log \frac{r^{*}}{\lambda_{s}}$ develops a logarithmic 
behaviour in the small- $r$ region with frequency $\left(\sqrt{2} r^{*}\right)^{-1} \simeq R^{-1}$ : is that perhaps a possible interpretation of the time nature of the variable $r$ in that region?

On the whole, we think, the above discussion shows - at a sort of "phenomenological" level - that the irregular solutions may indeed vehicle the particles' information from distances of order $R$ down to the string size, thus suggesting that the unitarity loss of the $\rho(0)=0$ model is due to the opening up of the gate to other worlds. However, due to the lack of the theoretical ingredients from string theory mentioned in the beginning, in the present formulation we have been unable to really assess the reliability of the above suggestion and to discuss the physical interpretation of the state(s), living at $\lambda_{s}$, which could be responsible for the singular contributions to the action investigated here and for their intriguing consequences.

\section{Conclusions}

To sum up, in our study of the improved eikonal model with subleading correction we have found that:

- The source particles, after the interaction, besides being deflected, suffer a time delay which can be interpreted as a shift in the light-cone variables (section 2);

- The shock-wave metric fields $h_{ \pm \pm}$generated by the source particles are also shifted in an analogous way, after the interaction described by the eikonal amplitude (section 3);

- The subleading contributions to the eikonal, represented by the (multi) H-diagram and by the rescattering terms, provide corrections to the scattering angle of the particles and to the shifts of the metric fields $h_{ \pm \pm}$and $\Phi$ (section 4 ). Due to rescattering, the large-distance shift of the particle fields agrees with the scattering-angle description based on the action.

In order to find an adequate form for the self-consistent metric which embodies all these features, we have to modify the ACV expression (4.21).

As far as the shock waves are concerned, the improved expressions are obtained from eq. (3.14) by replacing the leading profile function $a_{0}(\boldsymbol{x})$ with the full profile function $a(\boldsymbol{x})$ derived in section 4.3 and by accordingly shifting the location of the shock waves of the amount $\pm \pi R \Delta(\boldsymbol{x})$ as explained in section 4.4 .

In turn, the support $\Sigma$ of the transverse field $\Phi$ is also shifted from the region $x^{+} x^{-}>0$ to the two (past and future) disconnected regions

$$
\begin{aligned}
& \Sigma^{(-)} \equiv\left\{x: x^{+}<-\pi R \bar{\Delta}_{\phi}(\boldsymbol{x}), x^{-}<-\pi R \Delta_{\phi}(\boldsymbol{x})\right\} \\
& \Sigma^{(+)} \equiv\left\{x: x_{R}^{+}>+\pi R \bar{\Delta}_{\phi}\left(\boldsymbol{x}_{R}\right), x_{R}^{-}>+\pi R \Delta_{\phi}\left(\boldsymbol{x}_{R}\right)\right\}
\end{aligned}
$$

where in the region $\Sigma^{(+)}$after the interaction we have used the rotated coordinates of eq. (2.55). Equivalently, the characteristic function of $\Sigma=\Sigma^{(-)} \cup \Sigma^{(+)}$reads

$$
\chi(\Sigma)=\Theta\left(\left(-x^{+}-\pi R \bar{\Delta}_{\phi}\right)\left(-x^{-}-\pi R \Delta_{\phi}\right)\right)+\Theta\left(\left(x_{R}^{+}-\pi R \bar{\Delta}_{\phi}\right)\left(x_{R}^{-}-\pi R \Delta_{\phi}\right)\right) .
$$




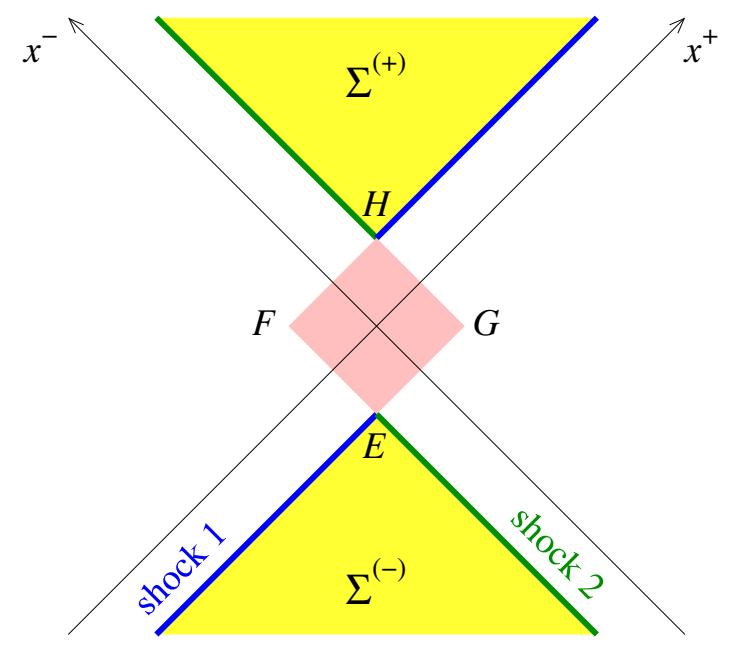

Figure 13. Longitudinal section of the space-time (at the transverse coordinate of the center of mass) showing the position of the shock waves 1 (blue) and 2 (green) and the support $\Sigma$ of the metric field $\Phi$ (yellow). The (pink) rectangle EFHG actually corresponds to two different regions of space-time, which are accessed either from the left or from the right.

The ensuing improved metric we propose is thus

$$
\begin{aligned}
\mathrm{d} s^{2}= & -\mathrm{d} x^{+} \mathrm{d} x^{-}\left[1-\frac{1}{2} \chi(\Sigma)(1-\dot{\rho})\right] \\
& +\left(\mathrm{d} x^{+}\right)^{2} \delta\left(x^{+}-\pi R \epsilon\left(x^{-}\right) \bar{\Delta}(\boldsymbol{x})\right)\left[2 \pi R \bar{a}(\boldsymbol{x})-\frac{1}{4}(1-\dot{\rho})\left|x^{-}-\pi R \epsilon\left(x^{+}\right) \Delta(\boldsymbol{x})\right|\right] \\
& +\left(\mathrm{d} x^{-}\right)^{2} \delta\left(x^{-}-\pi R \epsilon\left(x^{+}\right) \Delta(\boldsymbol{x})\right)\left[2 \pi R a(\boldsymbol{x})-\frac{1}{4}(1-\dot{\rho})\left|x^{+}-\pi R \epsilon\left(x^{-}\right) \bar{\Delta}(\boldsymbol{x})\right|\right] \\
& +\mathrm{d} r^{2}\left[1+2(\pi R)^{2} \chi(\Sigma) \dot{\phi}\right]+\mathrm{d} \theta^{2} r^{2}\left[1+2(\pi R)^{2} \chi(\Sigma)\left(\dot{\phi}+2 r^{2} \ddot{\phi}\right)\right] .
\end{aligned}
$$

It takes into account all the physics results following the analysis of the improved eikonal model and the ACV effective action, and is self-consistent, in the sense that the trajectory shifts caused by the shock-wave metric are felt by the metric itself.

A word of caution has to be spent concerning the form of the metric in eq. (6.3) in the region between the forward and past light-wedges. In fact, the set $\left|x^{+}\right|<\pi R \bar{\Delta},\left|x^{-}\right|<\pi R \Delta$ enclosed by the continuation of the shock waves - represented by a rectangle $E F H G$ for each longitudinal section at fixed $\boldsymbol{x}$ as depicted in figure 13 - actually represents two distinct physical regions in our coordinate system. Each region is unambiguously described by continuing the metric either from the left or from the right. For instance, by continuing the metric from the left across the boundary $E F H$, we enter the region at the left of both shocks, where shock 1 travels from $E$ to $G$, collides with shock 2 in $G$, and after that shock 2 moves from $G$ to $H$ and goes on in the $x^{-}$direction, as shown. On the other hand, either continuation leads to the same form of the metric inside the future light-wedge $\Sigma^{(+)}$.

We have also investigated here the scattering features of UV-sensitive solutions of the model in the subcritical range $\lambda_{s}<b, r \lesssim R$, without making a real attempt to restore the string degrees of freedom — which are nevertheless called for by the singular short-distance 
behaviour of the rescattering current. This preliminary analysis leads to the suggestion that such solutions may indeed carry the information from distances of order $R$ to distances of order $\lambda_{s}$, but a dynamical analysis in the string-dominated region is needed to find out about their fate.

\section{Acknowledgments}

It is a pleasure to thank Gabriele Veneziano for various exchanges on the topics presented here, and Domenico Seminara for useful discussions.

\section{A H-diagram insertions}

We start noticing that the field-insertions we shall consider are basically either (a) external (figure 10.a) or (b) internal (figure 10.b,c). Here we shall concentrate on the final external insertion kinematics which - in the frame characterized by initial particle's momenta $p_{1}+$ $q, p_{2}$ and final momenta $k, k_{2}$ - is able to provide the full shift $2 \pi R \Delta$ from past to future.

The field $\tilde{h}^{++}$is coupled to the charge $\kappa\left(k^{+}+q^{+}\right)\left(k^{+}\right)$and thus the insertion factor in front of the amplitude i $\mathcal{M}$ is

$$
\tilde{h}^{++}=\mathrm{i} \kappa \int \frac{\mathrm{d} q^{+} \mathrm{d} q^{-}}{4 \pi^{2}} \frac{k^{+} \mathrm{e}^{-\frac{\mathrm{i}}{2}\left(q^{-} x^{+}+q^{+} x^{-}\right)}}{\left[k^{-}+q^{-}-\frac{(\boldsymbol{k}+\boldsymbol{q})^{2}-\mathrm{i} \varepsilon}{k^{+}+q^{+}}\right]\left(q^{-}-\frac{\boldsymbol{q}^{2}-\mathrm{i} \varepsilon}{q^{+}}\right) q^{+}} \frac{\mathrm{d}^{2} \boldsymbol{q}}{(2 \pi)^{2}} \mathrm{e}^{\mathrm{i} \boldsymbol{q} \cdot \boldsymbol{x}}
$$

where $k^{+}+q^{+}$and $k^{+}$are both taken to the positive and larger than $\left|q^{+}\right|$. The value of $k^{-}=\boldsymbol{k}^{2} / k^{+}$is fixed by the mass-shell and the $q^{-}$-integration is done in the lower half-plane because $k^{+}+q^{+}>0$ picking up the pole at $q^{-}=\frac{(\boldsymbol{k}+\boldsymbol{q})^{2}}{k^{+}+q^{+}}-\frac{\boldsymbol{k}^{2}}{k^{+}}$. The result is

$$
\begin{aligned}
& \tilde{h}^{++}=\kappa \int \frac{\mathrm{d} q^{+}}{4 \pi} \mathrm{e}^{-\frac{\mathrm{i}}{2} q^{+} x^{-}}\left(k^{+}+q^{+}\right) \frac{\mathrm{d}^{2} \boldsymbol{q}}{2 \pi}\left[\frac{\Theta\left(x^{+}\right)}{\left(\boldsymbol{q}-\boldsymbol{k} \frac{q^{+}}{k^{+}}\right)^{2}}\left(\mathrm{e}^{-\frac{\mathrm{i}}{2} q^{-} x^{+}}-\Theta\left(q^{+}\right) \mathrm{e}^{-\frac{\mathrm{i}}{2} \frac{q^{2}}{q+} x^{+}}\right)\right. \\
& \left.+\Theta\left(-x^{+}\right) \frac{\Theta\left(-q^{+}\right) \mathrm{e}^{-\mathrm{i} \frac{q^{2}}{q^{+}} x^{+}}}{\left(\boldsymbol{q}-\boldsymbol{k}_{k^{+}}\right)^{q^{+}}}\right] \mathrm{e}^{\mathrm{i} \boldsymbol{q} \cdot \boldsymbol{x}}
\end{aligned}
$$

Here we have kept for completeness the soft-emission contribution at $q^{2}=0$ also which is however suppressed by the phase factor exponent $\boldsymbol{q}^{2} x^{+} / q^{+} \simeq x^{-} x^{+} / r^{2}$ in the fast-emitting particle kinematics we are using. The dominant contribution, due to the $(k+q)^{2}=0$ pole, has a small phase factor $q^{-} x^{+} \simeq x^{+} /(r b E) \ll 1$, and thus it provides the $\Theta\left(x^{+}\right)$result that we have anticipated in the text. ${ }^{18}$

Note also that the $q$-translation in the $\boldsymbol{q}^{2}$ denominator is due to the scattering angle implied by the $\boldsymbol{k}$ momentum transfer, which in a single-hit process is small, $\tan (\theta / 2) \simeq$ $|\boldsymbol{k}| / k^{+}=\mathcal{O}(1 /(\sqrt{s} b))$ and is thus negligible. On the other hand, in the full scattering

\footnotetext{
${ }^{18}$ Note, however, that in the $x^{+} \rightarrow 0^{+}$limit the whole contribution (A.1) vanishes for $q^{+}>0$, because the $q^{-}$-integrand has both poles in the same half-plane.
} 
process we have that $\sin (\theta / 2) \simeq Q / \sqrt{s}$ is sizeable, and the shock-wave is correspondingly rotated, as in eqs. (2.54), (3.14) of the text.

Therefore, for insertion on the eikonal lines in which $k^{+} \simeq p^{+}=\sqrt{s}$ and the amplitude factor is $2 \pi G s \mathrm{i} \mathcal{A}$, we recover the insertion factor

$$
h_{--}=\frac{\kappa}{2} \tilde{h}^{++}=2 \pi R\left(\mathrm{i} \sqrt{s}-2 \partial_{-}\right) \Theta\left(x^{+}\right) \delta\left(x^{-}\right) a_{0}(r)
$$

that we have repeatedly used in the text. Here $a_{0}$ is the leading profile function of eq. (2.38) and the possible rotation along the scattered beams is understood.

In the case of the H-diagram, besides the insertion (a) on external lines (already computed in the text) we have in principle two insertions, one of which is on the internal (eikonal) propagators. We think that this one (figure 10.b) is already included by the Lipatov's vertices, which by definition count the external particles' emissions also. Therefore, in order to avoid double-counting we only estimate here the insertion on the rescattering graviton propagator of momentum $k$ in figure 10.c, as emitted by Lipatov's vertices.

In order to do that, we compute the imaginary part of the $q$-insertion amplitude, which has the form

$$
\begin{aligned}
2 \Im \Delta h^{++}(x)=2 \pi G s R^{2} \int \mathrm{d}^{2} \boldsymbol{k} \frac{\mathrm{d}^{2} \boldsymbol{q}}{(2 \pi)^{2}} h^{*}(\boldsymbol{k}) h(\boldsymbol{k}+\boldsymbol{q}) \frac{\mathrm{d} k^{+} \mathrm{d} k^{-}}{8 \pi^{2}} \frac{\mathrm{d} q^{+} \mathrm{d} q^{-}}{4 \pi^{2}} \mathrm{e}^{-\frac{\mathrm{i}}{2}\left(q^{-} x^{+}+q^{+} x^{-}\right)} \mathrm{e}^{\mathrm{i} \boldsymbol{q} \cdot \boldsymbol{x}} \\
\quad \times 2 \pi \delta\left(k^{-}-\frac{\boldsymbol{k}^{2}}{k^{+}}\right)\left(k^{-}+q^{-}-\frac{(\boldsymbol{k}+\boldsymbol{q})^{2}-\mathrm{i} \varepsilon}{k^{+}+q^{+}}\right)^{-1}\left(q^{-}-\frac{\boldsymbol{q}^{2}-\mathrm{i} \varepsilon}{q^{+}}\right)^{-1}\left(q^{+}\right)^{-1} .
\end{aligned}
$$

By going through the same steps as before, we perform the $q^{-}$-integration first (by keeping the leading $(k+q)^{2}=0$ contribution only) and we have, in addition, a weighted $k^{+}, \boldsymbol{k}$-integration, as follows:

$$
\begin{aligned}
2 \Im \Delta h^{++}(x)=\kappa \Theta\left(x^{+}\right) G s R^{2} & \int \mathrm{d}^{2} \boldsymbol{k} \frac{\mathrm{d}^{2} \boldsymbol{q}}{2 \pi^{2}} h^{*}(\boldsymbol{k}) h(\boldsymbol{k}+\boldsymbol{q}) \\
& \times \int \frac{\mathrm{d} k^{+} \mathrm{d} q^{+}}{4 \pi} \frac{k^{+}+q^{+}}{2 k^{+}} \frac{\mathrm{e}^{\mathrm{i} \boldsymbol{q} \cdot \boldsymbol{x}}}{\left(\boldsymbol{q}-\boldsymbol{k}_{k^{+}}\right)^{2}} \mathrm{e}^{-\frac{1}{2} q^{+} x^{-}} .
\end{aligned}
$$

In the strong ordering region $\sqrt{s} \gg k^{+} \gg|\boldsymbol{k}| \sim 1 / b,\left|q^{+}\right|$we estimate the effect of the (small) $q$-translation (or $\boldsymbol{k}$-dependent rotation) by redefining $\tilde{\boldsymbol{q}}=\boldsymbol{q}-\boldsymbol{k} q^{+} / k^{+}$and neglecting the translation in the matrix element $h(\boldsymbol{k}+\boldsymbol{q})$. The integral in $\mathrm{d}^{2} \boldsymbol{q}$ becomes

$$
\int \frac{\mathrm{d}^{2} \tilde{\boldsymbol{q}}}{2 \pi^{2}} \frac{\mathrm{e}^{\mathrm{i} \tilde{\boldsymbol{q}} \cdot \boldsymbol{x}}}{\tilde{\boldsymbol{q}}^{2}} \mathrm{e}^{\mathrm{i} \boldsymbol{k} \cdot \boldsymbol{x} q^{+} / k^{+}} h^{*}(\boldsymbol{k}) h(\boldsymbol{k}+\tilde{\boldsymbol{q}})
$$

by thus factorizing the exponential $\mathrm{e}^{\mathrm{i} k \cdot \boldsymbol{x} q^{+} / k^{+}}$which modifies the subsequent $k^{+}$and $q^{+}$ integrations. By performing the latter in the phase-space $-q^{+}<k^{+}<\infty$, we obtain in turn the factor

$$
\simeq \mathrm{i} \partial_{-} \int_{|\boldsymbol{k}|}^{\sqrt{s}} \frac{\mathrm{d} k^{+}}{k^{+}}\left[\delta\left(x^{-}-2 \frac{\boldsymbol{k} \cdot \boldsymbol{x}}{k^{+}}\right)-\frac{1}{2 \pi} \frac{\mathrm{e}^{-\frac{k^{+}}{2}\left(\varepsilon-\mathrm{i}\left(x^{-}-2 \boldsymbol{k} \cdot \boldsymbol{x} / k^{+}\right)\right)}}{\varepsilon-\mathrm{i}\left(x^{-}-2 \frac{\boldsymbol{k} \cdot \boldsymbol{x}}{k^{+}}\right)}\right]
$$




$$
\simeq \log (\sqrt{s} b) \mathrm{i} \partial_{-} \delta\left(x^{-}\right) \times\left[1+\mathcal{O}\left(\frac{1}{\sqrt{s} b}\right)\right] .
$$

We can see here the small shock-wave rotation $\tan (\theta / 2) \simeq|\boldsymbol{k}| / k^{+}=\mathcal{O}(1 /(\sqrt{s} b)) \simeq \mathrm{e}^{-y}$ which has been neglected in front of the logarithmic phase space. By then collecting the various factors we obtain the result

$$
\Im \Delta h_{--}=\frac{\kappa}{2} \Im \Delta \tilde{h}^{++}=2 \pi R \Theta\left(x^{+}\right) \cdot 2 \delta^{\prime}\left(x^{-}\right) \cdot \pi R\left[G_{0} \Im a_{H}\right],
$$

where we have defined

$$
\begin{aligned}
{\left[G_{0} \Im a_{H}\right](\boldsymbol{x}) } & \equiv \pi R^{2} Y \int \mathrm{d}^{2} \boldsymbol{x}^{\prime}\left|\tilde{h}\left(\boldsymbol{x}^{\prime}\right)\right|^{2} G_{0}\left(\boldsymbol{x}-\boldsymbol{x}^{\prime}\right) \stackrel{r \geqq b}{\simeq} a_{0}(r) \cdot \Im a_{H}(b), \\
\Im a_{H} & =\frac{2 Y}{\pi} \Re a_{H} .
\end{aligned}
$$

This shift of the imaginary part and the corresponding one for the real part add up to the total shift $2 a_{H}$ of both real and imaginary parts due to the external line insertion

$$
\left.\Delta\left(\mathrm{i} A_{H}\right)\right|_{\mathrm{ext}}=2 \pi R \Theta\left(x^{+}\right) \pi R a_{H}\left(\mathrm{i} \sqrt{s}-4 \partial_{-}\right) \delta\left(x^{-}\right)
$$

and thus occurs at large distances only with a relative minus sign, as emphasized in the text.

Open Access. This article is distributed under the terms of the Creative Commons Attribution License (CC-BY 4.0), which permits any use, distribution and reproduction in any medium, provided the original author(s) and source are credited.

\section{References}

[1] D. Amati, M. Ciafaloni and G. Veneziano, Superstring collisions at planckian energies, Phys. Lett. B 197 (1987) 81 [InSPIRE].

[2] D. Amati, M. Ciafaloni and G. Veneziano, Classical and quantum gravity effects from planckian energy superstring collisions, Int. J. Mod. Phys. A 3 (1988) 1615 [INSPIRE].

[3] D. Amati, M. Ciafaloni and G. Veneziano, Can space-time be probed below the string size?, Phys. Lett. B 216 (1989) 41 [INSPIRE].

[4] D.J. Gross and P.F. Mende, The high-energy behavior of string scattering amplitudes, Phys. Lett. B 197 (1987) 129 [INSPIRE].

[5] D.J. Gross and P.F. Mende, String theory beyond the Planck scale, Nucl. Phys. B 303 (1988) 407 [inSPIRE].

[6] D. Amati, M. Ciafaloni and G. Veneziano, Higher order gravitational deflection and soft bremsstrahlung in planckian energy superstring collisions, Nucl. Phys. B 347 (1990) 550 [INSPIRE].

[7] D. Amati, M. Ciafaloni and G. Veneziano, Effective action and all order gravitational eikonal at planckian energies, Nucl. Phys. B 403 (1993) 707 [InSPIRE].

[8] D. Amati, M. Ciafaloni and G. Veneziano, Towards an S-matrix description of gravitational collapse, JHEP 02 (2008) 049 [arXiv: 0712.1209] [INSPIRE]. 
[9] M. Ciafaloni and D. Colferai, S-matrix and quantum tunneling in gravitational collapse, JHEP 11 (2008) 047 [arXiv:0807.2117] [INSPIRE].

[10] M. Ciafaloni and D. Colferai, Quantum tunneling and unitarity features of an S-matrix for gravitational collapse, JHEP 12 (2009) 062 [arXiv:0909.4523] [INSPIRE].

[11] P.C. Aichelburg and R.U. Sexl, On the gravitational field of a massless particle, Gen. Rel. Grav. 2 (1971) 303 [InSPIRE].

[12] A. Einstein, L. Infeld and B. Hoffmann, The gravitational equations and the problem of motion, Annals Math. 39 (1938) 65 [INSPIRE].

[13] A. Einstein and L. Infeld, The gravitational equations and the problem of motion. II, Annals Math. 41 (1940) 455 [INSPIRE].

[14] M. Ciafaloni, D. Colferai and G. Falcioni, Unitarity alternatives in the reduced-action model for gravitational collapse, JHEP 09 (2011) 044 [arXiv: 1106.5628] [INSPIRE].

[15] G. 't Hooft, Graviton dominance in ultrahigh-energy scattering, Phys. Lett. B 198 (1987) 61 [INSPIRE].

[16] D. Amati, M. Ciafaloni and G. Veneziano, private communication.

[17] L.N. Lipatov, High-energy scattering in QCD and in quantum gravity and two-dimensional field theories, Nucl. Phys. B 365 (1991) 614 [INSPIRE].

[18] R. Kirschner and L. Szymanowski, Effective action for high-energy scattering in gravity, Phys. Rev. D 52 (1995) 2333 [hep-th/9412087] [INSPIRE].

[19] L.N. Lipatov, Multi-Regge processes in gravitation, Sov. Phys. JETP 55 (1982) 582 [Zh. Eksp. Teor. Fiz. 82 (1982) 991] [INSPIRE].

[20] G. D'Appollonio, P. Di Vecchia, R. Russo and G. Veneziano, High-energy string-brane scattering: leading eikonal and beyond, JHEP 11 (2010) 100 [arXiv:1008.4773] [INSPIRE].

[21] S.B. Giddings, D.J. Gross and A. Maharana, Gravitational effects in ultrahigh-energy string scattering, Phys. Rev. D 77 (2008) 046001 [arXiv:0705.1816] [INSPIRE].

[22] V.S. Rychkov, private communication.

[23] M. Ciafaloni, D. Colferai and G. Veneziano, work in progress. 